\title{
Energy Efficient and Throughput Optimal CSMA Scheme
}

\author{
Ali Maatouk*, Mohamad Assaad*, and Anthony Ephremides ${ }^{\dagger}$ \\ *TCL Chair on 5G, Laboratoire des Signaux et Systèmes, CentraleSupélec, Gif-sur-Yvette, France \\ ${ }^{\dagger}$ ECE Dept., University of Maryland, College Park, MD 20742
}

\begin{abstract}
\Abstract-Carrier Sense Multiple Access (CSMA) is widely used as a Medium Access Control (MAC) in wireless networks due to its simplicity and distributed nature. This motivated researchers to find CSMA schemes that achieve throughput optimality. In 2008, it has been shown that a simple CSMA-type algorithm is able to achieve optimality in terms of throughput and has been given the name "adaptive" CSMA. Lately, new technologies emerged where prolonged battery life is crucial such as environment and industrial monitoring. This inspired the foundation of new CSMA based MAC schemes where links are allowed to transition into sleep mode to reduce the power consumption. However, throughput optimality of these schemes was not established. This paper therefore aims to find a new CSMA scheme that combines both throughput optimality and energy efficiency by adapting to the throughput and power consumption needs of each link. This is done by controlling operational parameters such as back-off and sleeping timers with the aim of optimizing a certain objective function. The resulting CSMA scheme is characterized by being asynchronous, completely distributed and being able to adapt to different power consumption profiles required by each link while still ensuring throughput optimality. The performance gain in terms of energy efficiency compared to the conventional adaptive CSMA scheme is demonstrated through computer simulations.
\end{abstract}

Index Terms-CSMA, Throughput Optimality, Energy Efficiency, IoT, Wireless MAC.

\section{INTRODUCTION}

$\mathrm{T}$ HROUGHPUT optimality for network scheduling, which is, by definition, the ability to withstand any arrival rates inside the capacity region of the network [1], has been thoroughly investigated in the literature. Tassiulas and Ephremides introduced in their seminal paper [2] the maximalweight scheduling (MWS), a throughput optimal scheduling scheme. Despite its provable optimality, this centralized scheme requires solving the maximum-weight independent set, which is an NP-Hard problem in general. With the aim of overcoming this complexity, a surge of papers have been published. The efforts were divided into two classes: the first one aimed to propose low complexity algorithms for certain interference models (e.g. [3] and the references therein) while the other focused on proposing low complexity algorithms that guarantee a portion of the capacity region [4] or a worstcase performance [5]. These approaches suffer from message passing, which can create a lot of congestion especially in the case of high links density.

This work has been performed in the framework of the Horizon 2020 project ONE5G (ICT-760809) receiving funds from the European Union.
Since the work on Max-Weight, it has been a long-standing open problem in the research community to find simple random access schemes that achieve full optimality for a general interference model without any message passing. It wasn't until 2008 that a simple capacity achieving Carrier Sense Multiple Access (CSMA) scheme, called the "adaptive CSMA", was introduced [1]. CSMA is a class of simple and distributed algorithms and is considered one of the most popular random access protocols. In this class of algorithms, a node senses the medium and transmits a packet only if the medium is sensed idle. A suboptimal CSMA scheme has been already adopted for practical applications (e.g. CSMA is the basic medium access algorithm in IEEE 802.11). Since the work of [1], more and more research interests in this socalled optimal CSMA area have been taken in the community [5]. For instance, the authors in [6] proposed a distributed queue length based CSMA protocol that was proven to be throughput optimal. Interestingly, none of the previous work on optimal CSMA have investigated the power consumption aspect of these schemes. In fact, the adaptive CSMA suffers from high energy consumption: when in back-off stages, continuous sensing of the environment by each link is required to spot any interfering transmissions from its neighbors which would result in an inevitable power consumption [1]. With long battery life being a strict requirement for emerging technologies, this line of work is of broad interest.

On the other hand, low power consumption has been the core focus in the design of Medium Access Control (MAC) protocols in Wireless Sensor Networks (WSN) [7]. These protocols mainly rely on the idea of duty cycling. Duty cycling is an effective method of reducing energy dissipation in Wireless Sensor Networks (WSNs) where a link is periodically placed into sleep mode. A large amount of MAC protocol solutions that are based on duty cycling were therefore proposed for WSN in the literature [7]. For instance, Sensor-MAC, one of the most famous low-energy MAC protocols, was proposed in [8]. It is based on sleep schedules that are managed by virtual clusters. Other MAC protocols were also introduced to further enhance the performance [9]. These protocols however suffer from message passing in order to maintain synchronization. WiseMac is another MAC protocol that was introduced to the WSN literature in [10]. In this protocol, all nodes wake up regularly using a common sleeping period but with different sleep schedule offsets. The clock drift can be compensated through several proposed methods (see, e.g., [11]). Although 
there have been a decent number of propositions, the throughput optimality of these proposed methods was not established.

The primary contribution of our paper is the introduction of a distributed CSMA scheme that does not include any message passing and combines both aformentioned aspects: throughput optimality and energy efficiency. The proposed scheme involves giving each link the freedom to transition between $A W A K E$ and SLEEP states. However, unlike the conventional duty cycling that has been previously proposed in the literature, the sleeping duration of each link is not fixed and is calibrated with the aim of optimizing a particular objective function. In fact, we argue that by jointly controlling both the sleeping and back-off duration of each link with the aim of optimizing a certain objective function, the power consumption of each link can be reduced while still maintaining the ability to withstand any feasible arrival rate. Our second and main contribution revolves around the use of different mathematical tools to prove vital theorems related to our scheme. In fact, the additional freedom given to each node will drastically change the CSMA model and the subsequent analysis as compared to the work in [1] as one will see in the sequel (e.g. the proof of theorem 2 is based on totally different tools). Consequently, after appropriate analysis, the result will be a fully distributed MAC algorithm in which time is not slotted (hence no synchronization required) and does not suffer from any message passing or clock drift issues. A new parameter will reveal itself which is assigned to each link based on a satisfactory power-delay tradeoff. Moreover, as a final contribution, we provide implementations insights on our scheme. We show that the proposed scheme, due to the dynamic nature of the activity of links, achieves similar collisions performance as the adaptive CSMA while drastically reducing the power consumption which makes it appealing to be implemented in practice. The performance gain with respect to the optimal adaptive CSMA scheme is corroborated by computer simulations.

The paper is organized as follows: Section II describes the system model and presents the proposed CSMA scheme. A Markov chain based analysis is provided in Section III to prove the throughput optimality of our proposed scheme and introduce the notion of energy efficiency. Insights on the implementation of our scheme in practical scenarios are given in Section IV. Section V provides numerical results that corroborate the theoretical results while Section VI concludes the paper.

\section{System Model And Proposed Algorithm}

\section{A. Proposed Scheme}

We consider a wireless network composed of $K$ links using the same bandwidth where each link is an (ordered) transmitter-receiver pair. In the sequel, we primarily focus on the transmitter side of each link in which we are interested in its ability to send DATA while carefully addressing its power consumption. In this framework, the receiver side of each pair can be a certain access point that is always ON (an application example being a Home Automation Network (HAN) [12]) or a device that is equipped with a radio-triggered wake-up mechanism [13]. Based on that, the words "link" and "transmitter" will be used interchangeably in the remainder of the paper. The proposed scheme in this paper belongs to the family of CSMA MAC protocols where transmitters in the network listen to the medium before proceeding to the transmission. More specifically, an active transmitter (i.e. not asleep) waits for a certain duration before transmitting, called the back-off time. While waiting, it keeps sensing the environment to spot any conflicting transmissions. If an interfering transmission is spotted, the transmitter stops immediately its back-off timer and waits for the medium to be free to resume it. Motivated by reducing the power consumption, we provide each transmitter the freedom to transition between two states:

- SLEEP state: in this state, power consumption is minor and no sensing of the environment takes place (the transmitter's radio is OFF)

- $A W A K E$ state: the transmitter's radio is $\mathrm{ON}$ and the adaptive CSMA scheme takes place as will be detailed in the sequel

The decision to either wake-up/sleep is dictated by an appropriate timer. When a transmitter decides to sleep, it picks an exponentially distributed wake-up time with mean $1 / W_{k}$ after which it wakes up. Once the transmitter is awake, it picks an exponentially chosen sleep timer with mean $1 / S_{k}$ after which it goes back to sleep. The motivation behind the exponential distribution assumption is the memory-less property that allows us to pursue a Markov chain based analysis as will be further explored in a future section. When the transmitter is awake, the adaptive CSMA scheme takes place:

- An exponentially distributed back-off timer with mean $1 / R_{k}$ is picked

- Continuous sensing of the channel takes place and whenever the channel is sensed idle, the back-off timer runs otherwise it is frozen. In both cases, the sleep timer keeps running

- Once the back-off timer runs out, the sleep timer is frozen and the packet's transmission starts.

- Packet's transmission time is assumed to be exponentially distributed with an average channel holding time $1 / H_{k}$

- After successful transmission, the sleep-timer is resumed and a new back-off timer is picked for the next transmission

\section{B. System Model}

In this section, we provide the necessary system model details to further proceed with the mathematical analysis. As a first step, we tackle the interference model. More precisely, the interference between the links is modeled by a conflict graph, which is a common model used in the literature and more precisely in the area of random access and CSMA based modeling. We recall that in the aim of reducing power consumption, we give the ability to each link to be either awake or asleep. In this context, we define the $j^{\text {th }}$ configuration state $a^{j}$ as a $K$-tuplet of binary variables $a_{k}^{j}$ that indicates if link $k$ is awake (binary value 1 ) or asleep (binary value 0 ). In fact, we have $2^{K}$ possible configuration states for the links. 
For each configuration state $a^{j}$, there exist a unique conflict graph $G_{j}=\left\{V_{j}, E_{j}\right\}$ where $V_{j}$ is the set of awake links and $E_{j}$ being the set of edges. Two vertices (awake links) have an edge between them if these two links cannot transmit at the same time. Clearly, not all links necessarily have the ability to transmit at the same time and we therefore define the notion of independent set of this conflict graph. Let $\left|I_{j}\right|$ be the total number of independent sets in the graph $G_{j}$, we denote the $i^{\text {th }}$ independent set as $x^{i}$. The independent set $x^{i}$ (also referred to as transmission state) is a $K$-tuplet of variables $x_{k}^{i}$ that indicates if link $k$ is transmitting or not. We assume that a link transmits just one packet when it acquires the channel, i.e., $x_{k}^{i}$ is a binary variable.

In the following, we adopt the standard idealized CSMA assumptions firstly introduced by [14] and adopted by the authors in [1]:

- The problem of hidden nodes does not exist

- Sensing is considered instantaneous, there is no sensing delay

The first condition is plausible in realistic scenarios if the range of carrier-sensing is large enough [15]. As for the second condition, it is violated in practical systems due to the finite speed of light and the time needed for a receiver to detect the radio signals. This condition however simplifies the mathematical model, making it tractable, which can act as a starting point before considering more complicated scenarios where this condition is violated (see Section IV-C for the case where this condition is violated). In addition to that, we assume that the links are always back-logged. With these idealized CSMA settings and the continuous nature of the back-off timer, collisions become mathematically impossible. This leads to tractability as a first step of the study and enables us to capture the essence of the scheduling problem without being concerned about the contention resolution problem (we tackle the contention resolution problem in Section IV-C). In the next section, we present a Markov chain based analysis of our scheme to prove its throughput optimality along with a discussion on its energy efficiency.

\section{MARKOV CHAIN BASED ANALYSIS}

\section{A. Proposed Markov Chain}

Let us consider the $2 \mathrm{D}$ continuous time stochastic process $\{(\boldsymbol{A}(t), \boldsymbol{X}(t)): t \geq 0\}$ where $\boldsymbol{A}(t) \in\{0,1\}^{K}$ and $\boldsymbol{X}(t) \in$ $\{0,1\}^{K}$ denote the configuration and transmission states of the network at time instant $t$ respectively. By adopting our proposed scheme, $\{(\boldsymbol{A}(t), \boldsymbol{X}(t)): t \geq 0\}$ becomes a Markovian process and the network can be therefore represented by a 2D continuous time Markov chain where each state in the chain is made of two components $\left(a^{j}, x^{i}\right)$ that were previously explained.

In the sequel, we define the transmission aggressiveness and the waking-up aggressiveness as $\boldsymbol{r} \in \mathbb{R}^{K}$ and $\boldsymbol{\rho} \in \mathbb{R}^{K}$ where $r_{k}=\log \left(R_{k} / H_{k}\right)$ and $\rho_{k}=\log \left(W_{k} / S_{k}\right)$ respectively.

Proposition 1. The 2D continuous time Markov chain is irreducible, time-reversible and is fully characterized by the transmission aggressiveness $\boldsymbol{r}$ and waking-up aggressiveness $\boldsymbol{\rho}$.
Moreover, this chain admits $\pi\left(a^{j}, x^{i} ; \boldsymbol{r}, \boldsymbol{\rho}\right)$ as stationary distribution for any feasible state $\left(a^{j}, x^{i}\right)$ (i.e. $\lim _{t \rightarrow \infty} P((\boldsymbol{A}(t)=$ $\left.\left.\left.a^{j}, \boldsymbol{X}(t)=x^{i}\right)\right)=\pi\left(a^{j}, x^{i} ; \boldsymbol{r}, \boldsymbol{\rho}\right)\right)$ where:

$$
\pi\left(a^{j}, x^{i} ; \boldsymbol{r}, \boldsymbol{\rho}\right)=\frac{\exp \left(\sum_{k=1}^{K} a_{k}^{j} \rho_{k}\right) \exp \left(\sum_{k=1}^{K} x_{k}^{i} r_{k}\right)}{C(\boldsymbol{r}, \boldsymbol{\rho})}
$$

and $C(\boldsymbol{r}, \boldsymbol{\rho})$ is a normalization factor:

$$
C(\boldsymbol{r}, \boldsymbol{\rho})=\sum_{j=1}^{2^{K}} \exp \left(\sum_{k=1}^{K} a_{k}^{j} \rho_{k}\right) \sum_{i=1}^{\left|I_{j}\right|} \exp \left(\sum_{k=1}^{K} x_{k}^{i} r_{k}\right)
$$

Proof: It is sufficient to show that the preceding distribution verifies the detailed balance equations [16].

Step 1: Consider the following two states $\left(a^{j}, x^{i}\right)$ and $\left(a^{j}+\right.$ $\left.\boldsymbol{e}_{\boldsymbol{k}}, x^{i}\right)$ where $a_{k}^{j}=0$ and $\boldsymbol{e}_{\boldsymbol{k}}$ represents the canonical vector in $\mathbb{R}^{K}\left(\left(\boldsymbol{e}_{\boldsymbol{k}}\right)_{l: 1 \leq l \leq K}=\left(\delta_{k l}\right)_{1 \leq l \leq K}\right.$ where $\delta_{k l}$ is the Kronecker delta function). It can be verified that:

$$
\frac{\pi\left(a^{j}+\boldsymbol{e}_{\boldsymbol{k}}, x^{i} ; \boldsymbol{r}, \boldsymbol{\rho}\right)}{\pi\left(a^{j}, x^{i} ; \boldsymbol{r}, \boldsymbol{\rho}\right)}=\exp \left(\rho_{k}\right)
$$

Step 2: Consider the two states $\left(a^{j}, x^{i}\right)$ and $\left(a^{j}, x^{i}+\boldsymbol{e}_{\boldsymbol{k}}\right)$ where $a_{k}^{j}=1$ (the link has to be awake to be able to transmit), $x_{k}^{i}=0$ and $x_{k^{\prime}}^{i}=0 \forall k^{\prime} \in N_{j}(k)$ where the neighboring set of link $k$ at configuration state $a^{j}$ is defined as $N_{j}(k)=\left\{k^{\prime}\right.$ : $\left.\left(k, k^{\prime}\right) \in E_{j}\right\}$. It can also be verified that:

$$
\frac{\pi\left(a^{j}, x^{i}+\boldsymbol{e}_{\boldsymbol{k}} ; \boldsymbol{r}, \boldsymbol{\rho}\right)}{\pi\left(a^{j}, x^{i} ; \boldsymbol{r}, \boldsymbol{\rho}\right)}=\exp \left(r_{k}\right)
$$

To further clarify the model, a simple example of two interfering links is taken in Fig. 1. The dashed lines partition the chain in 4 regions where in each, the $2 \mathrm{D}$ states share the same configuration state. In the first partition, $a^{1}=(0,0)$ and all links are asleep and the only feasible transmission state is $x^{1}=(0,0)$. As for the partition where $a^{2}=(1,0)$, only link 1 is awake and therefore the only possible transmission states are $x^{1}=(0,0)$ and $x^{2}=(1,0)$. It is completely symmetrical for the case of $a^{3}=(0,1)$. As for the final partition where $a^{4}=(1,1)$, both links are awake. Since the two links are interfering, the conflict graph in this partition is made of two vertices mapped to the awake links with an edge existing between them. The independent sets of this conflict graph are $x^{1}=(0,0), x^{2}=(1,0)$, and $x^{3}=(0,1)$. In other words, the transmission state $x^{4}=(1,1)$ is unfeasible.

Remark 1. One can clearly see how our proposed chain is much more general than the standard CSMA Markov chain by observing that the partition of the chain where $a^{j}=(1, \ldots, 1)$ is nothing but the CSMA Markov chain firstly introduced in [14] and adopted in [1].

Using the 2D Markov chain, we can determine several key performance indicators of our scheme. First, the throughput achieved by user $k$ is simply the amount of time the chain is 


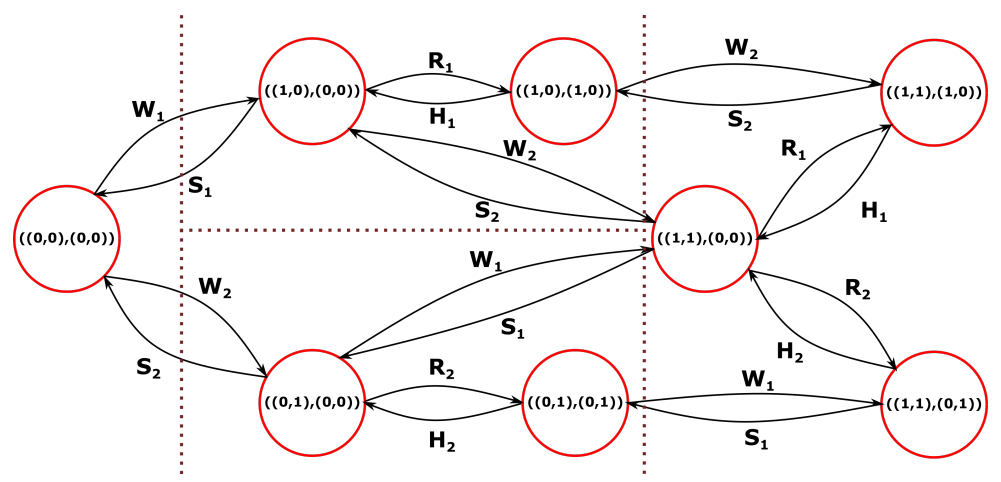

Fig. 1: Two interfering links Markov chain

in a state where link $k$ is both awake and transmitting. This can be expressed in the following way:

$$
s_{k}(\boldsymbol{r}, \boldsymbol{\rho})=\sum_{j=1}^{2^{K}} a_{k}^{j} \sum_{i=1}^{\left|I_{j}\right|} x_{k}^{i} \pi\left(a^{j}, x^{i} ; \boldsymbol{r}, \boldsymbol{\rho}\right)
$$

Moreover, we can define the awake duration of each link $k$ as the amount of time the chain is in a state where $a_{k}^{j}=1$. In other words:

$$
\hat{f}_{k}(\boldsymbol{r}, \boldsymbol{\rho})=\sum_{j=1}^{2^{K}} a_{k}^{j} \sum_{i=1}^{\left|I_{j}\right|} \pi\left(a^{j}, x^{i} ; \boldsymbol{r}, \boldsymbol{\rho}\right)
$$

\section{B. Optimality Analysis}

A scheduling scheme is said to be throughput optimal if it can support any feasible incoming rate $\lambda \in \mathbb{R}_{+}^{K}$, i.e., it stabilizes all the queues within the network whenever it is possible to do so. Knowing that packets arrive randomly with an average rate $\lambda_{k}$, the queue of link $k$ is defined as the number of packets buffered by the transmitter side of the link. A buffered packet leaves the system whenever link $k$ captures the channel for an exponential holding time of average $1 / H_{k}$. As it was shown, our scheme allows each link $k$ to achieve a throughput of $s_{k}(\boldsymbol{r}, \boldsymbol{\rho})$. We will be able to prove that our proposed scheme is indeed throughput optimal. However, before proceeding to our proof of throughput optimality, we first define the set of feasible rates $\Lambda$. Without loss of generality, we consider that the arrival rate vector $\boldsymbol{\lambda} \in \mathbb{R}_{+}^{K}$ is normalized to the capacity of each link, i.e. $\lambda_{k} \leq 1 \forall k$. In this case, $\lambda_{k}$ can be seen as the portion of time the chain should be in a state where user $k$ is both awake and transmitting to serve the arrival traffic. Hence, a rate is said to be feasible if it can be written as a joint probability distribution $\boldsymbol{p}$ over our Markov chain space. Therefore, for any feasible rate $\boldsymbol{\lambda}$, we have:

$$
\lambda_{k}=\sum_{j=1}^{2^{K}} \sum_{i=1}^{\left|I_{j}\right|} p_{i j} a_{k}^{j} x_{k}^{i}
$$

and $\boldsymbol{p}$ satisfies:

$$
p_{i j} \geqslant 0 \forall(i, j) \text { and } \sum_{j=1}^{2^{K}} \sum_{i=1}^{\left|I_{j}\right|} p_{i j}=1
$$

We can therefore define $\Lambda$ as follows:

$$
\Lambda=\left\{\boldsymbol{\lambda} \in \mathbb{R}_{+}^{K} \mid \exists \boldsymbol{p}: \lambda_{k}=\sum_{j=1}^{2^{K}} \sum_{i=1}^{\left|I_{j}\right|} p_{i j} a_{k}^{j} x_{k}^{i}\right\}
$$

where $\boldsymbol{p}$ verifies:

$$
\left\{\begin{array}{l}
p_{i j} \geqslant 0 \forall(i, j) \\
\sum_{j=1}^{2^{K}} \sum_{i=1}^{\left|I_{j}\right|} p_{i j}=1
\end{array}\right.
$$

Furthermore, we introduce a new parameter $f \in \mathbb{R}_{+}^{K}$, which we will refer to as the awake vector, as follows:

$$
f_{k}=\sum_{j=1}^{2^{K}} a_{k}^{j} \alpha_{j}=\mathbb{E}\left(a_{k}\right) \quad \text { and } \quad \alpha_{j}=\sum_{i=1}^{\left|I_{j}\right|} p_{i j}
$$

$\alpha_{j}$ represents the portion of time required for the network to be at configuration state $a^{j}$ while $f_{k}$ can be seen as the required awake duration of each link $k$, both as dictated by the arrival rate vector's joint probability distribution $p$. This newly introduced parameter will be key to the energy consumption aspect of our scheme as will be further detailed in the sequel. After these proper definitions, the throughput optimality of our proposed scheme is depicted in the following theorem.

Theorem 1. For any arrival rate $\boldsymbol{\lambda} \in \Lambda$, there exist $\left(\boldsymbol{r}^{*}, \boldsymbol{\rho}^{*}\right)$ such that $s_{k}\left(\boldsymbol{r}^{*}, \boldsymbol{\rho}^{*}\right)=\lambda_{k} \forall k$. Moreover, $\hat{f}_{k}\left(\boldsymbol{r}^{*}, \boldsymbol{\rho}^{*}\right)=f_{k} \forall k$.

Before proceeding to the proof, we first present our approach. For any feasible arrival rate $\lambda \in \Lambda$, our goal can be summarized as calibrating the parameters $(\boldsymbol{r}, \boldsymbol{\rho})$ in a way to make our CSMA Markov chain's stationary distribution as close as possible to $\boldsymbol{p}$. This is equivalent to reducing the distance between these two distributions. Different measures between probability distributions exist but in our paper we adopt the Kullback-Leibler divergence as in [1]. This approach was firstly introduced by the authors in [1] and was motivated by results on the theory of Markov random fields [17]. In fact, when minimizing the Kullback-Leibler divergence between a certain joint distribution and a product-form joint distribution, some particular marginal distributions induced by these two distributions are equal. This approach fits our framework since 
the joint distribution for us is nothing but $\boldsymbol{p}$ and the productform distribution is the stationary distribution of our proposed CSMA scheme depicted in Proposition 1

Remark 2. Although our throughput optimality's approach is similar to that of [1], the subsequent analysis is different due to the introduction of new parameters. In fact, the structure of the optimization problem leads us to introduce new variables in order to simplify the analysis through convex optimization tools. Moreover, the results on the finiteness of the optimal parameters in Theorem 2, which are pivotal to the work, will be based on different mathematical tools as it will be explained in the sequel.

Proof: To pursue our optimality analysis, we formulate the optimization problem as follows:

$\underset{\boldsymbol{r}, \boldsymbol{\rho}}{\operatorname{minimize}} D(\boldsymbol{p} \| \pi(\boldsymbol{r}, \boldsymbol{\rho}))=\sum_{j=1}^{2^{K}} \sum_{i=1}^{\left|I_{j}\right|} p_{i j} \log \left(\frac{p_{i j}}{\pi\left(a^{j}, x^{i} ; \boldsymbol{r}, \boldsymbol{\rho}\right)}\right)$

The objective function can be reduced through several steps:

$$
\begin{aligned}
& \sum_{j=1}^{2^{K}} \sum_{i=1}^{\left|I_{j}\right|} p_{i j} \log \left(\frac{p_{i j}}{\pi\left(a^{j}, x^{i} ; \boldsymbol{r}, \boldsymbol{\rho}\right)}\right)=\sum_{j=1}^{2^{K}} \sum_{i=1}^{\left|I_{j}\right|} p_{i j} \log \left(p_{i j}\right) \\
& -\sum_{j=1}^{2^{K}} \sum_{i=1}^{\left|I_{j}\right|} p_{i j} \log \left(\pi\left(a^{j}, x^{i} ; \boldsymbol{r}, \boldsymbol{\rho}\right)\right)=\sum_{j=1}^{2^{K}} \sum_{i=1}^{\left|I_{j}\right|} p_{i j} \log \left(p_{i j}\right) \\
& \quad-\sum_{j=1}^{2^{K}} \sum_{i=1}^{\left|I_{j}\right|} p_{i j}\left(\sum_{k=1}^{K} x_{k}^{i} r_{k}+\sum_{k=1}^{K} a_{k}^{j} \rho_{k}-\log (C(\boldsymbol{r}, \boldsymbol{\rho}))\right)
\end{aligned}
$$

The first term being independent of $(\boldsymbol{r}, \boldsymbol{\rho})$, the problem can be reformulated as minimizing the following function:

$$
\begin{aligned}
F(\boldsymbol{r}, \boldsymbol{\rho}) & =-\sum_{k=1}^{K} \sum_{j=1}^{2^{K}} \sum_{i=1}^{\left|I_{j}\right|} p_{i j} x_{k}^{i} r_{k}-\sum_{k=1}^{K} \sum_{j=1}^{2^{K}} \sum_{i=1}^{\left|I_{j}\right|} p_{i j} a_{k}^{j} \rho_{k} \\
& +\log \left(\sum_{j=1}^{2^{K}} \exp \left(\sum_{k=1}^{K} a_{k}^{j} \rho_{k}\right) \sum_{i=1}^{\left|I_{j}\right|} \exp \left(\sum_{k=1}^{K} x_{k}^{i} r_{k}\right)\right)
\end{aligned}
$$

The first term can be further reduced to an easier form since $x_{k}^{i}$ cannot be equal to 1 unless link $k$ is actually awake. Therefore, multiplying by the binary variable $a_{k}^{j}$ does not change the value of this term:

$$
\sum_{k=1}^{K} \sum_{j=1}^{2^{K}} \sum_{i=1}^{\left|I_{j}\right|} p_{i j} x_{k}^{i} r_{k}=\sum_{k=1}^{K} \sum_{j=1}^{2^{K}} \sum_{i=1}^{\left|I_{j}\right|} p_{i j} a_{k}^{j} x_{k}^{i} r_{k}=\sum_{k=1}^{K} \lambda_{k} r_{k}
$$

As for the second term, we have:

$$
\sum_{k=1}^{K} \sum_{j=1}^{2^{K}} \sum_{i=1}^{\left|I_{j}\right|} p_{i j} a_{k}^{j} \rho_{k}=\sum_{k=1}^{K} \sum_{j=1}^{2^{K}} a_{k}^{j} \rho_{k} \sum_{i=1}^{\left|I_{j}\right|} p_{i j}=\sum_{k=1}^{K} f_{k} \rho_{k}
$$

In order to solve this problem in a simple way using convex optimization tools, we introduce the new variables $t_{j i}$ in the following manner:

$$
\begin{array}{cl}
\underset{\boldsymbol{r}, \boldsymbol{\rho}, \boldsymbol{t}}{\operatorname{minimize}} & -\sum_{k=1}^{K} \lambda_{k} r_{k}-\sum_{k=1}^{K} f_{k} \rho_{k}+\log \left(\sum_{j=1}^{2^{K}} \sum_{i=1}^{\left|I_{j}\right|} \exp \left(t_{j i}\right)\right) \\
\text { subject to } & t_{j i}=\sum_{k=1}^{K} a_{k}^{j} \rho_{k}+\sum_{k=1}^{K} x_{k}^{i} r_{k} \\
j=1, \ldots, 2^{K} i=1, \ldots,\left|I_{j}\right| .
\end{array}
$$

The objective function is made of convex/linear functions in $(\boldsymbol{r}, \boldsymbol{\rho}, \boldsymbol{t})$ since the $\log$-sum-exponential function is a well known convex function [18]. On top of that, our equality constraints are linear and our transformed OP is indeed convex. Therefore, we can use the Lagrangian function and obtain the global optimal solution of our problem (7) by the Karush-Kuhn-Tucker (KKT) conditions that are necessary and sufficient in our case [18]. These conditions simplify to the Lagrange multipliers conditions due to the absence of any inequality constraints. The Lagrangian function is formulated as follows:

$$
\begin{gathered}
\mathcal{L}(\boldsymbol{r}, \boldsymbol{\rho}, \boldsymbol{T}, \boldsymbol{\mu})=\sum_{j=1}^{2^{K}} \sum_{i=1}^{\left|I_{j}\right|} \mu_{j i}\left(-t_{j i}+\sum_{k=1}^{K} a_{k}^{j} \rho_{k}+\sum_{k=1}^{K} x_{k}^{i} r_{k}\right) \\
-\sum_{k=1}^{K} \lambda_{k} r_{k}-\sum_{k=1}^{K} f_{k} \rho_{k}+\log \left(\sum_{j=1}^{2^{K}} \sum_{i=1}^{\left|I_{j}\right|} \exp \left(t_{j i}\right)\right)
\end{gathered}
$$

where $\mu_{j i}$ represents the dual variable corresponding to the $\{j i\}^{t h}$ constraint. At the optimal point, the following condition holds:

$$
\frac{\partial \mathcal{L}\left(\boldsymbol{r}^{*}, \boldsymbol{\rho}^{*}, \boldsymbol{T}^{*}, \boldsymbol{\mu}^{*}\right)}{\partial t_{j i}}=-\mu_{j i}^{*}+\frac{\exp \left(t_{j i}^{*}\right)}{C\left(\boldsymbol{r}^{*}, \boldsymbol{\rho}^{*}\right)}=0
$$

Moreover, since the optimal point has to satisfy the imposed constraint, we have the following:

$$
t_{j i}^{*}=\sum_{k=1}^{K} a_{k}^{j} \rho_{k}^{*}+\sum_{k=1}^{K} x_{k}^{i} r_{k}^{*}
$$

Combining the above two conditions, we can conclude that:

$$
\mu_{j i}^{*}=\frac{\exp \left(\sum_{k=1}^{K} a_{k}^{j} \rho_{k}^{*}\right) \exp \left(\sum_{k=1}^{K} x_{k}^{i} r_{k}^{*}\right)}{C\left(\boldsymbol{r}^{*}, \boldsymbol{\rho}^{*}\right)}=\pi\left(a^{j}, x^{i} ; \boldsymbol{r}^{*}, \boldsymbol{\rho}^{*}\right)
$$

which is nothing but the stationary distribution of our chain. Therefore, replacing our dual variable by the stationary distribution of our chain in the subsequent analysis take into account both the first order condition with respect to $t_{j i}$ and the feasibility condition. Furthermore, the following condition also holds:

$$
\frac{\partial \mathcal{L}\left(\boldsymbol{r}^{*}, \boldsymbol{\rho}^{*}, \boldsymbol{T}^{*}, \boldsymbol{\mu}^{*}\right)}{\partial \rho_{k}}=-f_{k}+\sum_{j=1}^{2^{K}} \sum_{i=1}^{\left|I_{j}\right|} \mu_{j i}^{*} a_{k}^{j}=0
$$

Using our previous conclusion on $\mu_{j i}^{*}, 11$ will lead to:

$$
-f_{k}+\sum_{j=1}^{2^{K}} a_{k}^{j} \sum_{i=1}^{\left|I_{j}\right|} \pi\left(a^{j}, x^{i} ; \boldsymbol{r}^{*}, \boldsymbol{\rho}^{*}\right)=0
$$


This means that at the optimal point, $\hat{f}_{k}\left(\boldsymbol{r}^{*}, \boldsymbol{\rho}^{*}\right)=f_{k} \forall k$ or in other words, link $k$ is awake just as needed to be. Lastly:

$$
\frac{\partial \mathcal{L}\left(\boldsymbol{r}^{*}, \boldsymbol{\rho}^{*}, \boldsymbol{T}^{*}, \boldsymbol{\mu}^{*}\right)}{\partial r_{k}}=-\lambda_{k}+\sum_{j=1}^{2^{K}} \sum_{i=1}^{\left|I_{j}\right|} \mu_{j i}^{*} x_{k}^{i}=0
$$

As it has been used before, multiplying by the binary variable $a_{k}^{j}$ does not change this value due to the fact that a link cannot transmit if it is not awake. Also, using the previous conclusions on $\mu_{j i}^{*},(13)$ becomes:

$$
-\lambda_{k}+\sum_{j=1}^{2^{K}} a_{k}^{j} \sum_{i=1}^{\left|I_{j}\right|} x_{k}^{i} \pi\left(a^{j}, x^{i} ; \boldsymbol{r}^{*}, \boldsymbol{\rho}^{*}\right)=0
$$

Hence, at the optimal point, we have $s_{k}\left(\boldsymbol{r}^{*}, \boldsymbol{\rho}^{*}\right)=\lambda_{k}$.

Corollary 1.1. Let $Q_{k}(t)$ represents the queue length of link $k$ at time $t$. For any arrival rate $\boldsymbol{\lambda} \in \Lambda$, there exist $\left(\boldsymbol{r}^{*}, \boldsymbol{\rho}^{*}\right)$ such that the queuing systems of all links are rate stable (i.e. $\left.\lim _{t \rightarrow \infty} \frac{Q_{k}(t)}{t} \rightarrow 0 \forall k\right)$.

Proof: As previously proven in Theorem 1, there exist $\left(\boldsymbol{r}^{*}, \boldsymbol{\rho}^{*}\right)$ such that $s_{k}\left(\boldsymbol{r}^{*}, \boldsymbol{\rho}^{*}\right)=\lambda_{k} \forall k$. This is a sufficient condition for rate stability of each link's queuing system [19. p.17].

Consequently, by minimizing (8), we can achieve queuing rate stability of all links in the network for any arrival rate $\lambda \in \Lambda$. Moreover, by minimizing (8), the required awake duration of each link $k$ is also achieved. As seen from the expression of (8), this optimization require the knowledge of the arrival vector $\boldsymbol{\lambda}$ and awake vector $\boldsymbol{f}$. This is cumbersome on the network and is unfeasible in practice. However, by observing equations (12) and (14), we can see that these conditions depend on local information of each link $k$. Therefore, the optimum $\left(\boldsymbol{r}^{*}, \boldsymbol{\rho}^{*}\right)$ can be achieved in a distributed manner using a simple gradient descent algorithm where each link $k$ updates its own operational parameters $r_{k}$ and $\rho_{k}$. Details concerning this implementation are presented in Section IV-B.

\section{Energy Efficiency}

To understand the energy efficiency aspect of our proposed scheme, it is vital to answer the following question: what does the arrival rate $\boldsymbol{\lambda}$ truly dictate concerning the configuration states?

To answer that, we can clearly see from the expression for $\lambda_{k}$, for any feasible arrival rate $\lambda$, that the arrival rate only dictates the portion of time where link $k$ is both awake and transmitting. The other states where link $k$ is either: awake and in a back-off stage or asleep, are irrelevant to the arrival rate and therefore the portion of time in which the chain is in those states presents for us a degree of freedom we can take advantage of. In other words, a feasible arrival rate $\lambda$ has different joint probability distribution $\boldsymbol{p}$ representations each of which leading to the same $\boldsymbol{\lambda}$ but with different awake vector $\boldsymbol{f}$. To see this more clearly, for a fixed arrival rate $\lambda$, it is straightforward that the minimal value of $f_{k}$ is $\lambda_{k}$. In this case, the joint probability distribution $\boldsymbol{p}$ verifies $p_{i j}=0$ where $a_{k}^{j}=1$ and $x_{k}^{i}=0$ (i.e. we should not be in a state where link $k$ is awake and not transmitting). Similarly, on the other extreme, the maximal value for $f_{k}$ is 1 . In this case, $\boldsymbol{p}$ verifies $p_{i j}=0$ where $a_{k}^{j}=0$ (i.e. states where link $k$ is asleep are forbidden). Therefore, the network is defined by two vectors $(\boldsymbol{\lambda}, \boldsymbol{f})$ instead of being solely defined by the arrival rate vector. Motivated by these results, we define what we will call the awake region as the awake vector feasibility region:

$$
\Theta(\boldsymbol{\lambda})=\left\{\boldsymbol{f} \in \mathbb{R}_{+}^{K}: \lambda_{k} \leq f_{k} \leq 1\right\}
$$

We can conclude that the network is characterized by two regions rather than one: the capacity region and the awake region. An example is presented in Fig. 2 and 3 for the case of two interfering links.

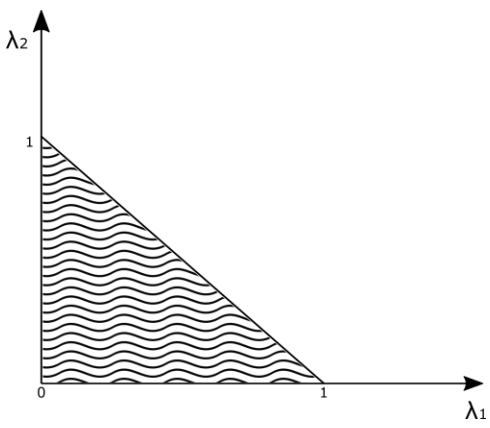

Fig. 2: Capacity region for the case of two interfering links

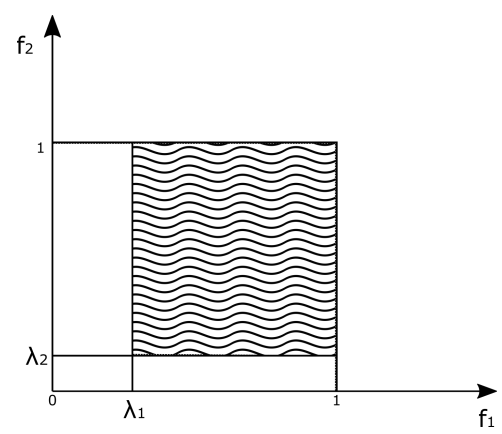

Fig. 3: Awake region for the case of two interfering links for a fixed $\boldsymbol{\lambda} \in \mathbb{R}_{+}^{2}$

After clarifying this, we can discuss the energy efficiency aspect of our proposed scheme. By rewriting $f_{k}$ in the following manner:

$$
f_{k}=\lambda_{k}+\omega_{k}
$$

where $0<\omega_{k}<1-\lambda_{k}$, we can see that a new parameter is born. This parameter is referred to as the Power-Delay Tradeoff (PDT) parameter and is assigned to each link $k$ as will be explained in the following. In fact, when we assign a small parameter value $\omega_{k}$ to link $k$, the required awake time $f_{k}$ will be close to $\lambda_{k}$. By recalling the results of Theorem 1 , we know that using our scheme, link $k$ will calibrate itself to satisfy the arrival and awake time requirements. Therefore, link $k$ will be awake just as necessary $\left(\hat{f}_{k}=f_{k} \approx \lambda_{k}\right.$ ) while still ensuring to satisfy its throughput requirement $\left(s_{k}=\lambda_{k}\right)$. To do so, link $k$ will spend a high amount of time being asleep but when it wakes up, it is extremely aggressive on the channel (small back-off timer) and therefore power consumption is reduced. However, due to the high fraction of time spent inactive, delays 


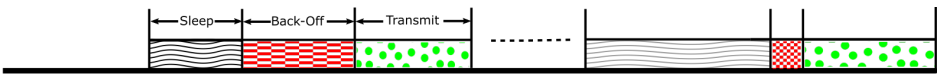

Link 2

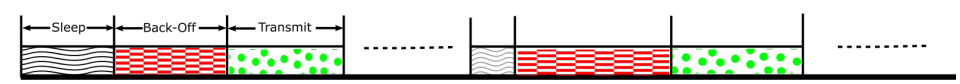

Fig. 4: Time-line of the proposed CSMA scheme

are to be anticipated which would manifest in long queues lengths. On the other extreme, if the assigned parameter $\omega_{k}$ to link $k$ is close to its maximal value, the required awake time $f_{k}$ will be close to 1 . In this manner, using our scheme, link $k$ will be forced to remain almost continuously awake while being less aggressive on the channe 1 Therefore, less delays would be expected but a higher power consumption takes place. In summary, $\omega_{k}$ is a Power-Delay Tradeoff (PDT) parameter that is assigned to each link $k$ depending on the desired tradeoff. The beauty of this parameter comes from the fact that IoT applications present a mixture of both delaysensitive and delay-tolerant applications. This can be exploited by simply calibrating the parameter assignment accordingly, the following table gives a few examples:

\begin{tabular}{|c|c|c|}
\hline \multicolumn{3}{|c|}{ IoT Applications } \\
\hline Category & Delay & Parameter $\omega_{k}$ \\
\hline Emergencies & Highly Sensitive & High \\
E-Health & Med. Sensitivity & Medium \\
Environment Monitors & Highly Tolerant & Low \\
\hline
\end{tabular}

TABLE I: Parameter Assignment

Remark 3. In the adaptive CSMA, the overall optimal transmission aggressiveness $\boldsymbol{r}^{*}$ (and therefore the optimal back-off timer) is just dictated by the network arrival rate vector $\lambda$ and the number of links in the collision domain. This might lead to relatively large back-off timers which would result in an inevitable power loss due to the continuous sensing of the environment. For our framework, we can work around this thanks to the newly introduced parameter. In fact, by assigning a small PDT parameter, links sleep to save power but are more aggressive on the channel once they are awake to compensate for the time spent in SLEEP state and achieve the required throughput. By doing so, back-off timers are drastically reduced (i.e. less power consumption from listening to the medium) and links therefore can still achieve throughput optimality even with their ability to transition to SLEEP state. For instance, one can see in Fig. 4 an example of the case of two links where link 1 is assigned a low PDT parameter unlike link 2 that is assigned a larger one. At first, both links start with the same initial waking-up and transmission aggressiveness $\left(\boldsymbol{r}_{0}, \boldsymbol{\rho}_{0}\right)$. As the parameters converge to their optimal values, link 1 ends up in SLEEP state for a large duration of time while being extremely aggressive on the network once it transitions to AWAKE state. On the other hand, link 2 is moderately aggressive on the network.

\footnotetext{
${ }^{1}$ The special case where $f_{k} \longrightarrow 1 \forall k$, all links will remain continuously awake and therefore the transition to SLEEP state does not take place for any link and we are back to the adaptive CSMA scheme in [1]
}

Remark 4. In general max-weight scheduling, the complexitydelay-stability tradeoff has been studied for wireless networks in the literature [20]. In our line of work, we are interested in CSMA based scheduling with the particularity to have energy consumption reduction while keeping the throughput optimality. The new parameter that we have just introduced $\boldsymbol{w}$, can be seen as a Power-Delay tradeoff parameter such that whatever the assigned parameter $\mathbf{0}<\boldsymbol{w}<\mathbf{1}-\boldsymbol{\lambda}$ is, the full stability region is achieved by the scheme.

This scheme will only be of interest if the optimum is always attained for a finite $\left(\boldsymbol{r}^{*}, \boldsymbol{\rho}^{*}\right)$. If this is not the case, the convergence to the optimal parameters $\left(\boldsymbol{r}^{*}, \boldsymbol{\rho}^{*}\right)$ does not take place and our scheme is unable to satisfy the throughput and awake duration requirements. This makes the results on the finiteness of the optimal parameters $\left(\boldsymbol{r}^{*}, \boldsymbol{\rho}^{*}\right)$ pivotal to our work. To prove this, the authors in [1] formulated a different optimization problem where the transmission aggressiveness is assumed to be positive and is taken as the dual variable of an inequality constraint in that new optimization problem. Consequently, they used the Slater's condition in that new OP as an initial step to proceed with the proof of finiteness. In our paper, we did not proceed with the same approach. In fact, forcing our new parameter to be positive will result in degradation in terms of energy efficiency and this was therefore omitted. To put this in perspective, suppose that we impose that $\rho_{k} \geq 0$, i.e. $\frac{W_{k}}{S_{k}} \geq 1$. We can see from this condition that we are forcing the ratio of the waking-up duration to the sleeping duration to be larger than 1 which is in complete contrast to the energy efficiency aspect of our scheme. This will be further highlighted later on in the simulations section. Therefore, we employed in the following theorem different machinery that helped us to prove the finiteness, baring in mind that we do not impose any conditions on our operational parameters.

Theorem 2. If $\boldsymbol{\lambda} \in \operatorname{int}(\Lambda)$ and $\boldsymbol{f} \in \operatorname{int}(\Theta(\boldsymbol{\lambda}))$, then the optimum is attained for a finite $\left(\boldsymbol{r}^{*}, \boldsymbol{\rho}^{*}\right)$

Proof: The proof can be found in the Appendix.

\section{IMPLEMENTATION CONSIDERATIONS}

\section{A. PDT Assignment}

The PDT parameter assignment can be either static (kept constant) or it can be dynamic by choosing an appropriate function of the backlog $Q_{k}$ of each link $k$.

First case: $\omega_{k}$ is assigned to each link and kept constant throughout transmission without change.

Second case: Once a large time frame $T_{c}$ (called convergence frame) has elapsed after which the transmission aggressiveness and wake-up aggressiveness attain their optimal value, a new 
constant $\omega_{k}=g_{k}\left(\bar{Q}_{k}\right)$ can be assigned to calibrate the powerdelay tradeoff based on the average backlog of link $k$ during $T_{c}$. By defining the stochastic backlog process of user $\mathrm{k}$ as $Q_{k}(\tau)$, the average backlog during the time frame $T_{c}$ can be calculated as follows:

$$
\bar{Q}_{k}=\frac{\int_{0}^{T_{c}} Q_{k}(\tau) d \tau}{T_{c}}
$$

This can be seen as a penalty function for the high queue length, that should be compensated in the next convergence frame. However this chosen function verifies certain properties, the first being that $g_{k}$ is an increasing function in $\bar{Q}_{k}$. Other properties include:

$$
0<g_{k}\left(\bar{Q}_{k}\right)<1-\lambda_{k} \quad \forall \bar{Q}_{k} \geq 0
$$

\section{B. Distributed Gradient Descent}

As previously discussed in Section III-B, queuing rate stability of all links in the network, for any arrival rate $\lambda \in \Lambda$, can be achieved by minimizing (8) through a distributed gradient algorithm. Moreover, the desired awake duration is achieved. Starting from an arbitrary point $\left(\boldsymbol{r}_{0}, \boldsymbol{\rho}_{0}\right)$, the updates of both the transmission and waking-up aggressiveness are done each time frame $T_{m}$ called update frame. However, due to the fact that the mixing time of the chain is slow [1], the updates should be done using sufficiently large time frames in order to ensure that the chain reaches its stationary distribution. Suppose that links update their aggressiveness at time $t_{m}$, we define $T_{m}=t_{m}-t_{m-1}$ as being the $m$-th update frame. The updates are consequently done as follow:

$$
\begin{gathered}
r_{k}(m+1)=r_{k}(m)+\triangle_{1 k}(m)\left(\lambda_{k}-s_{k}(m)\right) \\
\rho_{k}(m+1)=\rho_{k}(m)+\triangle_{2 k}(m)\left(\lambda_{k}+\omega_{k}-\hat{f}_{k}(m)\right)
\end{gathered}
$$

where $\triangle$ refers to the chosen step size. Both $s_{k}(m)^{2}$ and $\hat{f}_{k}(m)$ are calculated as follow:

$$
s_{k}(m)=\frac{\int_{t_{m}}^{t_{m+1}} a_{k}(\tau) x_{k}(\tau) d \tau}{T_{m+1}} \quad \hat{f}_{k}(m)=\frac{\int_{t_{m}}^{t_{m+1}} a_{k}(\tau) d \tau}{T_{m+1}}
$$

This process assumes that each link has knowledge of its arrival rate $\lambda_{k}$. However, if $\lambda_{k}$ is unknown, it can be approximated using its empirical average. In fact, by defining $V_{k}\left(T_{a}\right)$ as the total number of packet arrivals for link $k$ in the interval $\left[0, T_{a}\right]$, the arrival rate can be approximated by:

$$
\hat{\lambda}_{k}=\frac{V_{k}\left(T_{a}\right)}{T_{a}}
$$

with $T_{a}$ being sufficiently large since $\hat{\lambda}_{k} \rightarrow \lambda_{k}$ when $T_{a} \rightarrow$ $+\infty$.

Next, due to the fact that the updates create a fresh new Markov chain, residual effects from the previous chain should be mitigated. However, before proceeding, it is essential to point out an interesting aspect of our scheme. Knowing that

\footnotetext{
${ }^{2}$ We suppose that if the queue of link $k$ becomes empty, it sends dummy packets (i.e. the dummy packets are counted in the throughput computation of $s_{k}(m)$ in our gradient descent algorithm). This was done to comply with our scheme's assumption that aimed to simplify the Markov chain analysis. This simplification does not alter the stability region of the network (i.e. $s_{k}=\lambda_{k}$ ).
}

$\rho_{k}=\log \left(W_{k} / S_{k}\right)$, we can see that our scheme depends on the ratio of these two timers' means $\left(1 / S_{k}, 1 / W_{k}\right)$ and not individually on one of them. What this means is that we can choose $1 / S_{k}$ as small as desired to let link $k$ sleep quickly if it takes a long time to acquire the channel. This will make $1 / W_{k}$, for a fixed optimal $\boldsymbol{\rho}^{*}$, to be small as well to keep the same ratio. In other words, link $k$ will wake-up more often (shorter sleeping duration) to contend for the channel (in order to satisfy its throughput requirements) since its sleep timer is chosen to be small. On the other hand, if we choose $1 / S_{k}$ to be high, then we are forcing link $k$ to wait longer in its attempt to transmit data and not to directly transition into SLEEP state. This will result in having a larger $1 / W_{k}$ to maintain the same ratio: in other words, link $k$ will have to wake-up less often (longer sleeping duration) to satisfy its throughput requirement. This analysis has an interesting interpretation: the sleeping duration's mean of link $k\left(1 / W_{k}\right)$ calibrates itself to how patient we force link $k$ to be in order to still maintain the throughput optimality. In conclusion, although our proposed scheme seems at first in contrast to the line of work in WSN literature since links remain awake when the channel is sensed busy, the calibration property exhibited in our scheme makes it appealing in terms of energy consumption.

In the sequel, we will suppose that both $1 / S_{k}$ and $1 / H_{k}$ are fixed. In this case, to eliminate the residual effects from the previous iteration, the following take place:

- If the link was awake and transmitting when the update time frame has elapsed, the update of the parameters will not affect it and therefore no further actions are taken

- If the link was awake but in a back-off stage, a new back-off timer according to the new transmission aggressiveness should be chosen

- If the link was asleep, a new wake-up timer according to the new waking-up aggressiveness should be generated

\section{Contention Resolution}

We have so far focused in this paper on a continuous time CSMA model with no sensing delay. In these idealized settings, collisions are mathematically impossible, which leads to tractability as a first step of the study and enables us to capture the essence of the scheduling problem without being concerned about the contention resolution problem. However, in realistic scenarios, the sensing delay cannot be neglected and therefore the transmission back-off timer is chosen as a multiple of mini-slots where the duration of the mini-slot $T_{\text {slot }}$ is dictated by physical limitations such as propagation delay. In fact, once the wake-up timer of link $k$ ticks, link $k$ picks a random back-off timer uniformly distributed from the range $\left[0, W_{k}-1\right]$. In this case, the average back-off timer becomes $T_{\text {slot }} \frac{W_{k}-1}{2}$. We therefore suppose that the contention window $W_{k}$ of each link $k$ is chosen such that the mean back-off timer $1 / R_{k}$ is preserved which can be calculated from the transmission aggressiveness $r_{k}$. The back-off timer is decremented whenever the channel is sensed idle for a total mini-slot. In this case, collisions occur when at least two interfering links' back-off timers run down at the same time. Clearly, $100 \%$ throughput cannot be achieved in this 
case. However, as one will see in the sequel, we can achieve throughput close to the maximal allowed by the network. With this model under consideration, one has to compare its performance to the continuous counterpart that serves as a benchmark (i.e. we cannot achieve better performance than the continuous time version of our scheme). For this purpose, we present two distinct approaches:

Approach 1: Taking into account that $r_{k}=\log \left(R_{k} / H_{k}\right)$, we can rewrite the contention window's expression as follows:

$$
W_{k}=\frac{2}{\exp \left(r_{k}\right) \times H_{k} \times T_{\text {slot }}}+1
$$

In order to have a reasonably low collision probabilities, we seek to lowerbound the contention window of each link (i.e. $\left.W_{k} \geq W_{0} \forall k\right)$, conditioned on link $k$ being awake. In this setting, collisions can be ignored and the performance of the scheme (in both throughput and power consumption) virtually coincides with the continuous time collision-free scenario.

As it has been previously discussed, links that are assigned low PDT parameter tend to be more aggressive on the channel. Therefore, at first glance, one may think that assigning low PDT parameters to links will lead to an eventual high collisions probability due to the small contention window size as seen in (19). However, this is not the case due to the dynamic nature of links. In fact, although these links will be aggressive on the channel when they are awake, they spend a decent amount of time in SLEEP state which brings down the number of links in the collision domain. This will drastically reduce the collision probability resulting from the short back-off timers. As a matter of fact, it will be shown in the simulations section that we can achieve similar collision performance as the adaptive CSMA while still gaining in terms of power consumption. We can therefore conclude that it is vital to take into account the activity of the links when lowerbounding the contention window. For this purpose, we define what we will call the equivalent contention window as $\bar{W}_{k}=\frac{W_{k}}{1-P_{s_{k}}}$ where $P_{s_{k}}$ is the probability for link $k$ to be in SLEEP state. This can be thought as replacing link $k$ by link $k^{\prime}$ that stays awake continuously and therefore has an equivalent contention window of $W_{k^{\prime}}=\frac{W_{k}}{1-P_{s_{k}}}$. Hence, we shift our focus to lowerbounding the equivalent contention window of each link (i.e. $\bar{W}_{k} \geq W_{0} \forall k$ ).

To achieve this, first we assume that we are given a certain $T_{\text {slot }_{0}}$ and mean channel holding time $1 / H_{k}$. Next, to respect the required lower-bound, we are obliged to upperbound the transmission aggressiveness of each link $k$ by a certain value $r_{\max }$. Clearly, by doing so, the maximal throughput achieved by our scheme is reduced since we are limiting how aggressive links can be on the channel. However, as will be shown in the sequel, the performance degradation in realistic scenarios is minor. To see this more clearly, suppose we have $T_{\text {slot }}=9 \mu \mathrm{s}$ (as adopted in the IEEE 802.11n standard [21]), a mean channel holding time of $5 \mathrm{~ms}$ and a target contention window lowerbound $W_{0}=32$. This lowerbound leads to a reasonably low collision probability, assuming that the number of links in a collision domain is not too high (see [22] for further details). For instance, if links are kept continuously awake, we can see that to achieve the required lowerbound on the equivalent contention window, the transmission aggressiveness $r_{k}$ of each link $k$ should be upperbounded by $r_{\max }=3.5791 \forall k$. Although this upperbound seems small, it is actually able to achieve a high portion of the maximal throughput of the network. For illustration purposes, we suppose that we are in the case of two interfering links. In this scenario, the capacity region is defined as:

$$
\mathcal{C}=\left\{\boldsymbol{\lambda} \in \mathbb{R}_{+}^{2}: \lambda_{1}+\lambda_{2} \leq 1\right\}
$$

By keeping links continuously awake (i.e. by assigning the PDT parameter $\boldsymbol{w}$ to its its maximal value $\boldsymbol{w}_{\max }=\mathbf{1}-\boldsymbol{\lambda}$ ), the maximum allowed transmission aggressiveness is $r_{\max }=$ 3.5791. When $r_{1}=r_{2}=r_{\max }=3.5791$, we are able to achieve a total throughput of $s_{1}+s_{2}=0.986 \approx 1$ which is really close to the maximal throughput of 1 . As for smaller PDT assignments, the following table is presented ${ }^{3}$

\begin{tabular}{|c|c|c|}
\hline Parameter Assignment & $r_{\max }$ & Maximal Throughput \\
\hline $\boldsymbol{w}=(\mathbf{1}-\boldsymbol{\lambda})$ & 3.579 & 0.986 \\
$\boldsymbol{w}=(\mathbf{1}-\boldsymbol{\lambda}) / 2$ & 3.884 & 0.980 \\
$\boldsymbol{w}=(\mathbf{1}-\boldsymbol{\lambda}) / 4$ & 4.091 & 0.965 \\
$\boldsymbol{w}=(\mathbf{1}-\boldsymbol{\lambda}) / 8$ & 4.230 & 0.945 \\
\hline
\end{tabular}

TABLE II: Maximal Throughput Achieved

These results suggest that even for extremely low parameter assignments (which translates into a high power gain), we are still able to achieve decent performance, even when collisions are taken into accoun $4^{4}$. Moreover, we can achieve even higher throughput by increasing the mean channel holding time of each link $k$ since, for a given $W_{0}$, it will make $r_{\max }$ increase. Approach 2: In this approach, we recall that in order to achieve throughputs under slotted CSMA algorithms close to those obtained under the continuous-time CSMA algorithm, it is sufficient to keep the collision duration $T_{\text {collision }}$ small with respect to the channel holding time (as presented and tested in [23]). To accomplish this in our framework, we can adopt a variant of our scheme that takes into account the discrete nature of the back-off stage and approaches the optimal performance of the CSMA scheme. When the backoff timer of link $k$ runs out, the link probes the channel with a small signaling message of duration $\delta$ similar to the RTS/CTS mechanism adopted in IEEE 802.11. In this case, when a collision takes place, only these small signaling messages collide and therefore the collision duration is limited to $\delta$. By having an average transmission time $1 / H_{k}$ large compared to $\delta$, the throughputs achieved by each link are close to those of the continuous time counterpart. Of course, the incorporation of the RTS/CTS mechanism increases the overhead of successful transmissions. However, with this overhead being small compared to the overall transmission time, this mechanism comes at a fairly small penalty in terms of overall throughput $s_{k} \forall k$ and awake duration $f_{k} \forall k$ (and therefore power consumption)

\footnotetext{
${ }^{3}$ In this framework, we are not limiting the waking-up aggresiveness to a certain value. Therefore, the same performance in terms of power consumption, is to be anticipated. The only performance difference with respect to the benchmark lays in the throughput

${ }^{4}$ For instance, in the last table entry, $\boldsymbol{\lambda}=(0.4725,0.4725)$ and the parameter assignment is $\boldsymbol{w}=(0.0659,0.0659)$ which is considerably low and therefore would achieve high reduction in power consumption
} 
and the performance would virtually coincide with thus of the continuous time counterpart.

\section{Simulations}

The goal of these simulations is to corroborate the theoretical results in terms of parameters convergence and highlight the power gain experienced by the links using our proposed scheme in comparison to the adaptive CSMA counterpart. Moreover, the provided simulations put into perspective how the IEEE 802.11 protocol fails as the load on the network increases. Before proceeding to the simulations, we first present the power model taken into consideration:

- When the link is in a SLEEP state, the link consumes $P_{z}$

- When the link is transmitting, it consumes $P_{t}$

- When the link is sensing the medium, it consumes $P_{s}$. The link essentially receives radio signals and after signal processing, it takes a decision whether the medium is to be considered busy or not. We neglect the processing power consumption and assume that the power consumption in this case is simply the power required to receive these radio signals (i.e. $P_{s}=P_{r}$ )

As for the numerical values, we will adopt the values of CC1101 in Table III], a low power RF transceiver proposed by the industry [24]. It is worth mentioning that using the parameters of other low power transceivers yields similar relative results.

\begin{tabular}{|c|c|}
\hline \multicolumn{2}{|c|}{ Power Model } \\
\hline Power Parameter & Value \\
\hline$P_{z}$ & $1.5 \mu \mathrm{W}$ \\
$P_{t}$ & $73 \mathrm{~mW}$ \\
$P_{r}$ & $45 \mathrm{~mW}$ \\
\hline
\end{tabular}

TABLE III: Power model values

We assume that the channel mean holding time is $1 \mathrm{~ms}$, and as mentioned before, we are going to assume that the sleeping timer's mean is kept constant throughout the simulations, which is fixed to $1 \mathrm{~ms}$ in the sequel. We consider a realistic heterogeneous case where several groups of links exist in the network with each group having its own desired PDT. The number of groups is chosen as 3 with 4 links in each group: -Group 1: This group is made of links that are delay sensitive but can tolerate a high power consumption

-Group 2: This group is made of links that fall between the two extremes, they require a moderate power consumption without introducing a lot of delay

-Group 3: This group is made of links that can tolerate long delays however they are extremely power limited

We consider that the arrival for each link is $\lambda_{k}=0.077 \forall k$. $\omega_{1}=0.8, \omega_{2}=0.4$ and $\omega_{3}=0.1$ are assigned for Groups 1,2 and 3 respectively. The simulations are run for $100 s$ with a fixed update time frame $T_{m}=10 \mathrm{~ms}$ and with the same step size $\triangle=0.1$ for all updates.

Parameters Convergence: By solving our optimization problem previously stated in our theoretical analysis (see Section III-B), the optimal aggressiveness parameters can be found in the following table 5

\begin{tabular}{|c|c|c|c|}
\hline \multicolumn{4}{|c|}{ Optimization Problem Solutions } \\
\hline \multicolumn{3}{|c|}{ Proposed Scheme } & Adaptive CSMA \\
\hline Group Index & $r^{*}$ & $\rho^{*}$ & $r^{*}$ \\
\hline 1 & 0.1561 & 1.8724 & 0.014 \\
2 & 0.8492 & -0.2681 & 0.014 \\
3 & 2.2355 & -2.1078 & 0.014 \\
\hline
\end{tabular}

TABLE IV: Optimal Aggressiveness

It can be seen from these values that by adapting our scheme, links are more aggressive on the channel when they are awake compared to the adaptive CSMA (which translates into smaller back-off timers). Moreover, links with low powerdelay tradeoff parameter (i.e. links that spend more time in SLEEP state) are more aggressive on the channel when they wake-up. The extra aggressiveness can be thought to be a compensation for the time spent in SLEEP state. In fact, to be able to maintain their ability to withstand their arrival rate, links that spend a high amount of time in SLEEP state have to be aggressive on the channel to rapidly capture it. As demonstrated in both Fig. 5 and 6 , the optimum parameters are achieved distributively by each link, simply by monitoring:

1) the amount of time it spends being awake

2) the amount of time it spends transmitting $7^{7}$

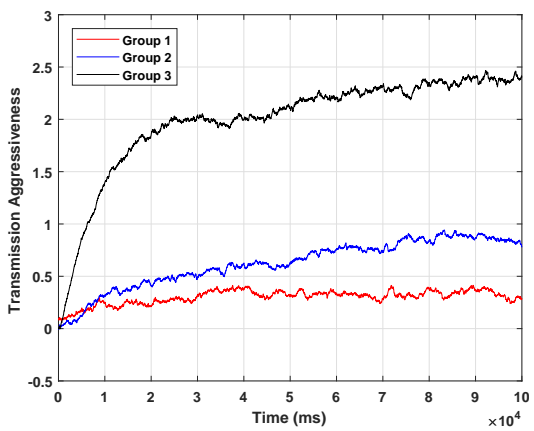

Fig. 5: Evolution of the transmission aggressiveness

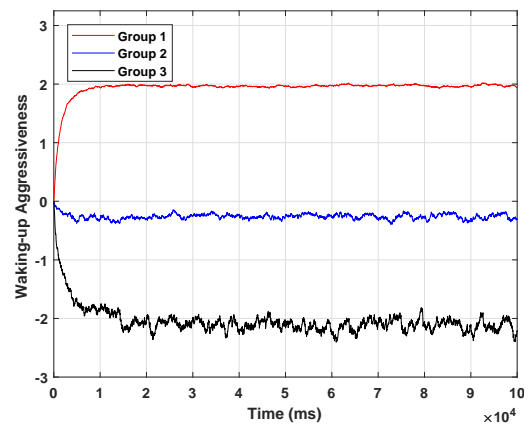

Fig. 6: Evolution of the waking-up aggressiveness

\footnotetext{
${ }^{5}$ Links within the same group share the same optimal aggressiveness parameters

${ }^{6}$ If we have imposed positivity of $\rho$, links of group 2 and 3 will be forced to stay awake longer and the performance in terms of energy efficiency will be hugely degraded.

${ }^{7}$ For ease of presentation, we have only shown the parameters convergence for an arbitrary link in each group while noting that links in each group experienced the same behavior
} 


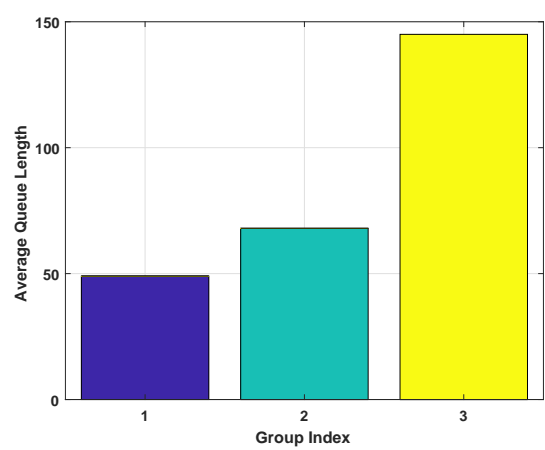

(a) Average queue length of each group

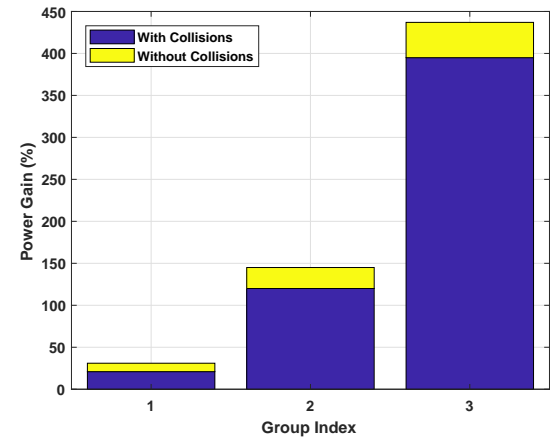

(b) Comparison with the adaptive CSMA in(c) Comparison in terms of successfully transterms of energy

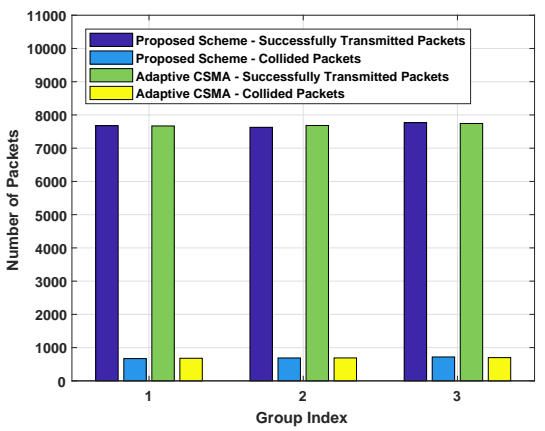

mitted and collided packets

Fig. 7: Simulations Results

Energy Efficiency: In order to best demonstrate the difference in performance between our scheme and the standard adaptive CSMA, we let the initial aggressiveness parameters to be close to their optimal values for both schemes.

The results are presented in both Fig $7 \mathrm{a}$ and Fig $7 \mathrm{~b}$ (without collisions) where the power is evaluated per successfully transmitted packet. As expected, Group 3 experienced the highest queue length but benefited from a high power gain (recall that links in Group 3 are delay tolerant). Group 1 on the other extreme had the complete opposite results while Group 2 laid in between these two cases. Fig $7 \mathrm{~b}$ shows the gain in terms of power of our scheme as compared to the adaptive CSMA. Our results show clearly that our scheme can handle multiple services at the same time by simply adjusting the PDT parameter. For some IoT applications (with delay tolerance), our scheme provides a huge power gain.

The effect of collisions: In this scenario, we relax the assumption of zero sensing delay. Therefore, collisions cannot be ignored any longer and a performance degradation with respect to the continuous time counterpart is to be expected. The mini-slot is chosen to be $T_{\text {slot }}=9 \mu$ s (as adopted in the IEEE 802.11n standard [21]). While taking into account the collisions, the following performance indicators are investigated:

1) Total number of successfully transmitted packets

2) Power gain with respect to the theoretical (collision-free) continuous time adaptive CSMA

We can see from the results in Fig. $7 \mathrm{~b}$ and $7 \mathrm{c}$ that although the arrival rate $\lambda$ is close to the capacity region's boundary $\left(\sum_{k=1}^{12} \lambda_{k}=0.924\right.$ close to the maximal throughput of 1$)$ and the collision domain's density is high (12 links in a single collision domain), we are still able to achieve performance close to the collision-free performance (a small degradation in terms of power gain is witnessed, which is natural due to the power lost on collided packets). Moreover, although links are aggressive on the channel using our scheme (especially those with low PDT parameters), we can see that the ratio of the lost packets to the total transmitted packets (i.e. the collision probability) is similar to the adaptive CSMA counterpart. This comes from the dynamic nature of the activity of links. In other words, although these links are aggressive on the channel, they spend a decent amount in SLEEP state which reduces the probability of collisions with other links. In summary, the performance of our scheme is close to the theoretical performance due to several factors:

1) The network consists of several groups with different degree of aggressiveness on the channel (e.g. some links are more aggressive on the channel than the others)

2) The dynamic nature of the activity of links: although several links are aggressive, they may be in SLEEP state when other links are contending for the channel

Comparison with IEEE 802.11: To further highlight the advantages of our proposed scheme, we compare it with IEEE 802.11 (Wi-Fi) as well. The configuration settings of IEEE 802.11 were set as follow:

- Binary exponential backoff is used with a maximum of 10 multiplications (i.e. $\mathrm{CW}_{\max }=1024 \times \mathrm{CW}_{0}$ )

- The contention window $\mathrm{CW}_{0}$ is set based on the work of Bianchi to achieve the highest possible throughput [22]

We can see in Fig. 8 that IEEE 802.11 is able to achieve a maximum throughput of around $s_{k}=0.068 \forall k$ (i.e the maximal total throughput is $\sum_{k=1}^{12} s_{k}=0.816$ ). This means that when $\lambda_{k}=0.077 \forall k$, the queue length of each link will grow indefinitely when IEEE 802.11 is used. This comes from the fact that IEEE 802.11 suffers from being throughput suboptimal [1]. On the other hand, one can clearly see in Fig. $7 \mathrm{c}$ that both the adaptive CSMA and our proposed scheme satisfy the requirements in terms of throughput $s_{k} \approx \lambda_{k}$ (each link sent $N$ successfully packets where $N \approx 0.077 \times 10^{5}=$ 7700 ). With our proposed scheme being throughput optimal, one can therefore expect an increase of the maximal possible throughput of around $20 \%$ with respect to IEEE 802.11. As for the power consumption, IEEE 802.11 experienced a $15 \%$ loss of power with respect to the adaptive CSMA. To conclude, our proposed scheme is able to satisfy the throughput requirements of links in high load environments due to its throughput optimality while providing huge power gain with respect to the adaptive CSMA.

\section{CONCLUSION}

In this paper, we have introduced a new MAC scheme that belongs to the CSMA family. In this scheme, with the aim of reducing power consumption, each link is allowed to transition between AWAKE and SLEEP states. By controlling operational parameters such as back-off and sleeping timers with the aim of optimizing a certain objective function, we 


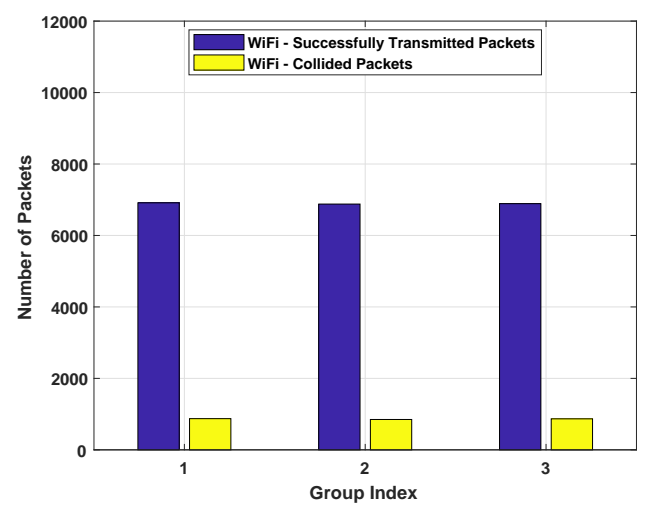

Fig. 8: IEEE 802.11 performance in terms of successfully transmitted and collided packets

were able to show that our scheme is throughput optimal. The convergence of the parameters to their optimal values has been proven to be completely distributed without any message passing. The theoretical analysis resulted in the birth of a parameter which had the interpretation of being a power-delay tradeoff. This parameter is assigned to each link depending on the application concerned. Implementation considerations were provided and the simulations conformed with the theoretical results and showed the performance advantages in terms of power gain with respect to the adaptive CSMA. In the future, the authors will focus on the study of the convergence of the proposed CSMA scheme along with a careful investigation of the convergence speed.

\section{ACKNOWLEDGMENTS}

The authors would like to express their gratitude to the Associate Editor and the reviewers for their many constructive comments and suggestions to improve the quality of the paper.

\section{REFERENCES}

[1] L. Jiang and J. Walrand, "A Distributed CSMA Algorithm for Throughput and Utility Maximization in Wireless Networks," IEEE/ACM Transactions on Networking, vol. 18, no. 3, pp. 960-972, June 2010.

[2] L. Tassiulas and A. Ephremides, "Stability properties of constrained queueing systems and scheduling policies for maximum throughput in multihop radio networks," IEEE Transactions on Automatic Control, vol. 37, no. 12, pp. 1936-1948, Dec 1992.

[3] A. Eryilmaz, A. Ozdaglar, and E. Modiano, "Polynomial Complexity Algorithms for Full Utilization of Multi-Hop Wireless Networks," in IEEE INFOCOM 2007 - 26th IEEE International Conference on Computer Communications, May 2007, pp. 499-507.

[4] X. Wu, R. Srikant, and J. R. Perkins, "Scheduling Efficiency of Distributed Greedy Scheduling Algorithms in Wireless Networks," IEEE Transactions on Mobile Computing, vol. 6, no. 6, pp. 595-605, June 2007.

[5] S. Y. Yun, Y. Yi, J. Shin, and D. Y. Eun, "Optimal CSMA: A survey," in 2012 IEEE International Conference on Communication Systems (ICCS), Nov 2012, pp. 199-204.

[6] J. Ni, B. Tan, and R. Srikant, "Q-CSMA: Queue-Length-Based CSMA/CA Algorithms for Achieving Maximum Throughput and Low Delay in Wireless Networks," IEEE/ACM Transactions on Networking, vol. 20, no. 3, pp. 825-836, June 2012.

[7] I. Demirkol, C. Ersoy, and F. Alagoz, "MAC protocols for wireless sensor networks: a survey," IEEE Communications Magazine, vol. 44, no. 4, pp. 115-121, April 2006.

[8] W. Ye, J. Heidemann, and D. Estrin, "An energy-efficient MAC protocol for wireless sensor networks," in Proceedings.Twenty-First Annual Joint Conference of the IEEE Computer and Communications Societies, vol. 3, 2002, pp. $1567-1576$ vol.3.
[9] T. van Dam and K. Langendoen, "An Adaptive Energy-efficient MAC Protocol for Wireless Sensor Networks," in Proceedings of the 1st International Conference on Embedded Networked Sensor Systems, ser. SenSys '03. New York, NY, USA: ACM, 2003, pp. 171-180. [Online]. Available: http://doi.acm.org/10.1145/958491.958512

[10] A. El-Hoiydi and J. D. Decotignie, "WiseMAC: an ultra low power MAC protocol for the downlink of infrastructure wireless sensor networks," in Proceedings. ISCC 2004. Ninth International Symposium on Computers And Communications (IEEE Cat. No.04TH8769), vol. 1, June 2004, pp. 244-251 Vol.1.

[11] B. Sundararaman, U. Buy, and A. D. Kshemkalyani, "Clock synchronization for wireless sensor networks: a survey," Ad Hoc Networks, vol. 3, no. 3, pp. 281 - 323, 2005. [Online]. Available: http://www.sciencedirect.com/science/article/pii/S1570870505000144

[12] G. M. Toschi, L. B. Campos, and C. E. Cugnasca, "Home automation networks: A survey," Computer Standards and Interfaces, vol. 50, pp. 42 - 54, 2017. [Online]. Available: http://www.sciencedirect.com/ science/article/pii/S0920548916300654

[13] L. Gu and J. A. Stankovic, "Radio-triggered wake-up capability for sensor networks," in Proceedings. RTAS 2004. 10th IEEE Real-Time and Embedded Technology and Applications Symposium, 2004., May 2004, pp. 27-36.

[14] R. Boorstyn, A. Kershenbaum, B. Maglaris, and V. Sahin, "Throughput analysis in multihop csma packet radio networks," IEEE Transactions on Communications, vol. 35, no. 3, pp. 267-274, Mar 1987.

[15] L. B. Jiang and S. C. Liew, "Improving throughput and fairness by reducing exposed and hidden nodes in 802.11 networks," IEEE Transactions on Mobile Computing, vol. 7, no. 1, pp. 34-49, Jan 2008.

[16] J. R. Norris, Markov Chains, ser. Cambridge Series in Statistical and Probabilistic Mathematics. Cambridge University Press, 1997.

[17] M. J. Wainwright and M. I. Jordan, "Graphical models, exponential families, and variational inference," Found. Trends Mach. Learn., vol. 1, no. 1-2, pp. 1-305, Jan. 2008. [Online]. Available: http: //dx.doi.org/10.1561/2200000001

[18] S. Boyd and L. Vandenberghe, Convex Optimization. New York, NY, USA: Cambridge University Press, 2004

[19] M. J. Neely, Stochastic Network Optimization with Application to Communication and Queueing Systems. Morgan and Claypool Publishers, 2010.

[20] Y. Yi, A. Proutière, and M. Chiang, "Complexity in wireless scheduling: Impact and tradeoffs," in Proceedings of the 9th ACM International Symposium on Mobile Ad Hoc Networking and Computing, ser. MobiHoc '08. New York, NY, USA: ACM, 2008, pp. 33-42. [Online]. Available: http://doi.acm.org/10.1145/1374618.1374624

[21] "IEEE Standard for Information technology- Local and metropolitan area networks- Specific requirements- Part 11: Wireless LAN Medium Access Control (MAC)and Physical Layer (PHY) Specifications Amendment 5: Enhancements for Higher Throughput," IEEE Std 802.11n-2009 (Amendment to IEEE Std 802.11-2007 as amended by IEEE Std 802.11 k2008, IEEE Std 802.11r-2008, IEEE Std 802.11y-2008, and IEEE Std 802.11w-2009), pp. 1-565, Oct 2009.

[22] G. Bianchi, "Performance analysis of the IEEE 802.11 distributed coordination function," IEEE Journal on Selected Areas in Communications, vol. 18, no. 3, pp. 535-547, March 2000.

[23] J. Liu, Y. Yi, A. Proutiere, M. Chiang, and H. V. Poor, "Convergence and tradeoff of utility-optimal CSMA," in 2009 Sixth International Conference on Broadband Communications, Networks, and Systems, Sept 2009, pp. 1-8.

[24] Texas Instruments, "CC1101 Low-Power Sub-1GHz RF Transceiver," http://www.ti.com/lit/ds/symlink/cc1101.pdf datasheet, Nov 2013.

\section{APPENDIX}

\section{PROOF OF THEOREM 2}

We recall that our original optimization problem is to minimize the Kullback-Leibler divergence between the joint probabiblity distribution $\boldsymbol{p}$ and our Markov chain's stationary distribution. Knowing that $D(\boldsymbol{p} \| \pi(\boldsymbol{r}, \boldsymbol{\rho})) \geqslant 0$, we can conclude:

$$
\inf _{\boldsymbol{r}, \boldsymbol{\rho}} D(\boldsymbol{p} \| \pi(\boldsymbol{r}, \boldsymbol{\rho})) \text { exists }
$$

Now what remains is to show that the minimum is attained by a finite $\left(\boldsymbol{r}^{*}, \boldsymbol{\rho}^{*}\right)$. The motivation behind this theorem's assumptions are the following: 
- if $f_{k}=\lambda_{k}$ : the only way to achieve this is for link $k$ to wake-up and instantly acquire the channel. Consequently for this case, the value of $r_{k}$ should tend to $+\infty$

- if $f_{k}=1$ : the only way to achieve this is for link $k$ to continuously stay awake. Consequently for this case, the value of $\rho_{k}$ should tend to $+\infty$

- As for the strict feasibility ${ }^{8}$ of $\lambda$ : when the arrival rate belongs to the boundary of the capacity region, links become extremely aggressive since the network hits its limit and consequently $r_{k} \forall k$ should tend to $+\infty$.

We provide a rigorous proof to our claim in the sequel. The proof is divided into two sections: first, a lemma is provided to show the equivalence between the assumptions of the theorem and the strict positivity of the joint probability distribution $p$ where:

$$
\left\{\begin{array}{l}
\lambda_{k}=\sum_{j=1}^{2^{K}} \sum_{i=1}^{\left|I_{j}\right|} p_{i j} a_{k}^{j} x_{k}^{i} \\
f_{k}=\sum_{j=1}^{2^{K}} \sum_{i=1}^{\left|I_{j}\right|} p_{i j} a_{k}^{j}
\end{array}\right.
$$

The results of this Lemma will be used to proceed with the proof by contradiction and making use of a fundamental mathematical theorem on bounded series: the "Bolzano-Weierstrass Theorem". In fact, the proof by contradiction assumes that $p_{i j}>0 \forall(i, j)$ which makes the results of this Lemma pivotal to the rest of the proof.

Lemma 2.1. The joint probability distribution $\boldsymbol{p}$ satisfies $p_{i j}>$ $0 \forall i, j$ if and only if

$$
\left\{\begin{array}{l}
\lambda_{k}>0 \forall k \\
\lambda \text { is strictly feasible } \\
\lambda_{k}<f_{k}<1 \forall k
\end{array}\right.
$$

Proof: The proof of this Lemma is divided into two parts where, in each part, one of the required implications is proven. Part 1: We start by proving that if $p_{i j}>0 \forall i, j$ then:

$$
\left\{\begin{array}{l}
\lambda_{k}>0 \forall k \\
\lambda \text { is strictly feasible } \\
\lambda_{k}<f_{k}<1 \forall k
\end{array}\right.
$$

- $\boldsymbol{f}<\mathbf{1}$ : the state $S=(\mathbf{0}, \mathbf{0})$ has a non-null probability then no link stay awake all the time and therefore we have $f_{k}<1 \forall k$

- $\boldsymbol{f}>\boldsymbol{\lambda}$ : states where links are awake and not transmitting have non zero probability i.e. links enter in back-off stages and therefore spend more time awake than transmitting

$-\boldsymbol{\lambda}>\mathbf{0}$ : it is straightforward since each link has an arrival rate of at least $p_{k}=\left(a^{j}=\boldsymbol{e}_{\boldsymbol{k}}, x^{i}=\boldsymbol{e}_{\boldsymbol{k}}\right)>0$

- Strict feasibility : we will have to prove that there is exist a neighborhood of $\boldsymbol{\lambda}$ such that each arrival rate inside of it is feasible. Let $p_{0}=P((\mathbf{1}, \mathbf{0}))>0$ the portion of time users are all awake and none of them is transmitting and $p_{k}=$ $P\left(\left(\mathbf{1}, \boldsymbol{e}_{\boldsymbol{k}}\right)\right)>0$ where $\boldsymbol{e}_{\boldsymbol{k}}$ refers to the $k^{\text {th }}$ canonical vector,

\footnotetext{
${ }^{8} \mathrm{~A}$ rate $\boldsymbol{\lambda}$ is said to be strictly feasible if $\boldsymbol{\lambda} \in \operatorname{int}(\Lambda)$ or in other words if there exist a neighborhood of $\boldsymbol{\lambda}$ such that every $\boldsymbol{\lambda}_{\boldsymbol{I}}$ that belongs to this neighborhood is feasible
}

we define $\epsilon=\min \left\{p_{0} / K, \min _{k} p_{k}\right\}>0$. We can satisfy any arrival rate $\lambda^{\prime}$ such that:

$$
\left|\lambda_{k}^{\prime}-\lambda_{k}\right| \leqslant \epsilon \forall k
$$

We can do that by simply constructing the following distribution $\boldsymbol{p}^{\prime}$ such that:

$$
\left\{\begin{array}{l}
p_{0}^{\prime}=p_{0}-\sum_{k=1}^{K}\left(\lambda_{k}^{\prime}-\lambda_{k}\right) \\
p_{k}^{\prime}=p_{k}+\lambda_{k}^{\prime}-\lambda_{k} \forall k \\
p^{\prime}=p \text { for all other states }
\end{array}\right.
$$

To note that in this case, $f_{k}^{\prime}=f_{k} \forall k$.

Part 2: Throughout this section, a visualization of the proof in the case of two interfering links is presented to clarify the explanation. In this part, we suppose that $\lambda$ and $f$ verify:

$$
\left\{\begin{array}{l}
\lambda_{k}>0 \forall k \\
\lambda \text { is strictly feasible } \\
\lambda_{k}<f_{k}<1 \forall k
\end{array}\right.
$$

We will try to construct a joint probability distribution $\boldsymbol{p}$ that satisfies $p_{i j}>0 \forall i, j$ such that:

$$
\left\{\begin{array}{l}
\lambda_{k}=\sum_{j=1}^{2^{K}} \sum_{i=1}^{\left|I_{j}\right|} p_{i j} a_{k}^{j} x_{k}^{i} \\
f_{k}=\sum_{j=1}^{2^{K}} \sum_{i=1}^{\left|I_{j}\right|} p_{i j} a_{k}^{j}
\end{array}\right.
$$

We start by choosing an arbitrary probability distribution $\boldsymbol{p}_{\boldsymbol{A}}>\mathbf{0}$, then four cases can occur:

(a): the randomly constructed $\boldsymbol{p}_{\boldsymbol{A}}$ verifies the requirements $\boldsymbol{\lambda}_{\boldsymbol{A}}=\boldsymbol{\lambda}$ and $\boldsymbol{f}_{\boldsymbol{A}}=\boldsymbol{f}$ then we choose $\boldsymbol{p}=\boldsymbol{p}_{\boldsymbol{A}}$

(b): $\boldsymbol{p}_{\boldsymbol{A}}$ verifies $\boldsymbol{\lambda}_{\boldsymbol{A}}=\boldsymbol{\lambda}$ but $\boldsymbol{f}_{\boldsymbol{A}} \neq \boldsymbol{f}$. Since both $\boldsymbol{f}_{\boldsymbol{A}}, \boldsymbol{f}>\boldsymbol{\lambda}$ (for $\boldsymbol{f}_{\boldsymbol{A}}$, we recall part 1 since $\boldsymbol{p}_{\boldsymbol{A}}>\mathbf{0}$ ) hence they lay in the same region and we can find $d>0$ such that:

$$
\boldsymbol{f}_{\boldsymbol{B}}=\boldsymbol{f}+d\left(\boldsymbol{f}-\boldsymbol{f}_{\boldsymbol{A}}\right)
$$

Therefore we can find $\boldsymbol{p}_{\boldsymbol{B}} \geqslant 0$ such that $\boldsymbol{\lambda}_{\boldsymbol{B}}=\boldsymbol{\lambda}$. We can then construct in this case $\boldsymbol{p}$ in the following way:

$$
p_{i j}=\theta p_{A_{i j}}+(1-\theta) p_{B_{i j}}
$$

where $\theta=\frac{d}{1+d}>0$. We know that $p_{A_{i j}}>0$ and $p_{B_{i j}} \geqslant 0$, therefore $p_{i j}>0$.

(c): $p_{A}$ verifies the requirement for $\boldsymbol{f}_{\boldsymbol{A}}=\boldsymbol{f}$ but $\boldsymbol{\lambda}_{\boldsymbol{A}} \neq \lambda$. It is tricky here since the region of $f$ depends on $\lambda$. In other words, $f_{\boldsymbol{A}}$ and $\boldsymbol{f}$ do not lay in the same region. However since $\boldsymbol{\lambda}<\boldsymbol{f}$, we can always find a neighborhood of $\boldsymbol{\lambda}$ such that for each $\lambda_{I}$ in it, $\lambda_{I}$ verifies $\lambda_{I}<f$. Now we take the intersections between this neighborhood and all the affine combinations between $\boldsymbol{\lambda}$ and $\boldsymbol{\lambda}_{A}$, then we can state that there is exist $\boldsymbol{\lambda}_{\boldsymbol{B}}$ in this intersection (hence verifies $\boldsymbol{\lambda}_{\boldsymbol{B}}<\boldsymbol{f}$ ) such that $\exists d>0$ in a way that:

$$
\boldsymbol{\lambda}_{\boldsymbol{B}}=\boldsymbol{\lambda}+d\left(\boldsymbol{\lambda}-\boldsymbol{\lambda}_{\boldsymbol{A}}\right)
$$

Let $\boldsymbol{p}_{B} \geqslant 0$ its corresponding probability distribution with $\boldsymbol{f}_{\boldsymbol{B}}=\boldsymbol{f}$ which is possible since $\boldsymbol{\lambda}_{\boldsymbol{B}}<\boldsymbol{f}$. Then we can 
conclude that we can write $\boldsymbol{p}$ in the following way:

$$
p_{i j}=\theta p_{A_{i j}}+(1-\theta) p_{B_{i j}}
$$

where $\theta=\frac{d}{1+d}>0$. We know that $p_{A_{i j}}>0$ and $p_{B_{i j}} \geqslant 0$, therefore $p_{i j}>0$. (d): in this case we have $\boldsymbol{f}_{\boldsymbol{A}} \neq \boldsymbol{f}$ and $\lambda_{\boldsymbol{A}} \neq \boldsymbol{\lambda}$. This might seems like the hardest case but it is merely a combination of both cases (b) and (c). Since the priority is to coincide the regions of $\boldsymbol{f}_{\boldsymbol{A}}$ and $\boldsymbol{f}$, we start by using the same analysis as case (c). By doing so, we have:

$$
\boldsymbol{\lambda}_{\boldsymbol{B}}=\boldsymbol{\lambda}+d_{1}\left(\boldsymbol{\lambda}-\boldsymbol{\lambda}_{\boldsymbol{A}}\right)
$$

and let $\boldsymbol{p}_{B} \geqslant 0$ be its corresponding probability distribution with $f_{B}=f$. We construct the probability distribution $p_{C}$ as follows:

$$
p_{C_{i j}}=\theta_{1} p_{A_{i j}}+\left(1-\theta_{1}\right) p_{B_{i j}}
$$

where $\theta_{1}=\frac{d_{1}}{1+d_{1}}>0$. We know that $p_{A_{i j}}>0$ and $p_{B_{i j}} \geqslant 0$, we can conclude that $p_{C_{i j}}>0$. We now have $\boldsymbol{\lambda}_{C}=\boldsymbol{\lambda}$ but $f_{C} \neq f$. We are back to case (2) and therefore, we can find $d_{2}>0$ such that:

$$
\boldsymbol{f}_{\boldsymbol{D}}=\boldsymbol{f}+d_{2}\left(\boldsymbol{f}-\boldsymbol{f}_{\boldsymbol{C}}\right)
$$

and $\boldsymbol{\lambda}_{\boldsymbol{D}}=\boldsymbol{\lambda}$. We can then construct in this case $\boldsymbol{p}$ :

$$
p_{i j}=\theta_{2} p_{C_{i j}}+\left(1-\theta_{2}\right) p_{D_{i j}}
$$

where $\theta_{2}=\frac{d_{2}}{1+d_{2}}>0$. Knowing that $p_{C_{i j}}>0$ and $p_{D_{i j}} \geqslant 0$, we have $p_{i j}>0$ which concludes the proof of the Lemma

The next step is to use the results of Lemma. 2.1 to prove that our optimum is attained for a finite $\left(r^{*}, \rho^{*}\right)$. As proven in Lemma. 2.1. our theorem's assumptions are equivalent to supposing that $p_{i j}>0 \forall(i, j)$. Taking that into consideration, we consider in the following that $p_{i j}>0 \forall i, j$. First, we recall our optimization problem:

$$
\underset{\boldsymbol{r}, \boldsymbol{\rho}}{\operatorname{minimize}} F(\boldsymbol{r}, \boldsymbol{\rho})=-\sum_{j=1}^{2^{K}} \sum_{i=1}^{\left|I_{j}\right|} p_{i j} \log \left(\pi\left(a^{j}, x^{i} ; \boldsymbol{r}, \boldsymbol{\rho}\right)\right)
$$

We have previously proven in Section III-B that this minimization is indeed a convex optimization problem after appropriate transformations. Therefore, we either have one of the following cases:

(a): a finite minimizer $\left(\boldsymbol{r}^{*}, \boldsymbol{\rho}^{*}\right)$ exists and it is unique due to the convexity of our optimization problem

(b): there exist a sequence $\left(\boldsymbol{r}^{n}\right)_{n}$ such that $F\left(\left(\boldsymbol{r}^{n}\right)_{n}, \boldsymbol{\rho}^{*}\right) \stackrel{n \rightarrow+\infty}{\longrightarrow} F^{*}$ and $\left\|\boldsymbol{r}^{n}\right\| \stackrel{n \rightarrow+\infty}{\longrightarrow}+\infty$

(c): there exist a sequence $\left(\rho^{n}\right)_{n}$ such that $F\left(\boldsymbol{r}^{*},\left(\boldsymbol{\rho}^{n}\right)_{n}\right) \stackrel{n \rightarrow+\infty}{\longrightarrow} F^{*}$ and $\left\|\boldsymbol{\rho}^{n}\right\| \stackrel{n \rightarrow+\infty}{\longrightarrow}+\infty$

(d): there exist two sequences $\left(\boldsymbol{r}^{n}\right)_{n}$ and $\left(\boldsymbol{\rho}^{m}\right)_{m}$ such that $F\left(\left(\boldsymbol{r}^{n}\right)_{n},\left(\boldsymbol{\rho}^{m}\right)_{m}\right) \stackrel{n, m \rightarrow+\infty}{\longrightarrow} F^{*}$ while the norm of these two vectors $\left\|\boldsymbol{r}^{n}\right\|,\left\|\boldsymbol{\rho}^{m}\right\| \stackrel{n, m \rightarrow+\infty}{\longrightarrow}+\infty$.

The main idea revolves around proving that case (a) is the only possible outcome of the optimization problem. For this purpose, we recall a fundamental mathematical theorem on bounded sequences. Before stating the theorem, we call to mind the notion of accumulation point. Let $\left(\boldsymbol{a}_{n}\right)$ be a sequence of real vectors, the vector $\boldsymbol{L}$ is said to be an accumulation point of $\left(\boldsymbol{a}_{n}\right)$ if there exists a subsequence $\left(\boldsymbol{a}_{n_{k}}\right)$ that converges to $\boldsymbol{L}$. In other words:

$$
\forall \epsilon>0, \exists K \in \mathbb{N} \text { such that if } k \geqslant K \text { then }\left\|\boldsymbol{a}_{n_{k}}-L\right\| \leqslant \epsilon
$$

Theorem 3 (Bolzano-Weierstrass Theorem). Each bounded sequence in $\mathbb{R}^{K}$ has at least one accumulation point or equivalently at least one convergent subsequence.

Armed with this theorem, we proceed with our proof by contradiction. Consider that case (b) occurs, we can rewrite the sequence $\boldsymbol{r}^{n}$ as follows: $\boldsymbol{r}^{n}=\left\|\boldsymbol{r}^{n}\right\| \frac{\boldsymbol{r}^{n}}{\left\|\boldsymbol{r}^{n}\right\|}$ where $\frac{\boldsymbol{r}^{n}}{\left\|\boldsymbol{r}^{n}\right\|}$ is a bounded sequence. Knowing that $\frac{r^{n}}{\left\|r^{n}\right\|}$ in $\mathbb{R}^{K}$ is a bounded sequence, the theorem states that $\frac{\boldsymbol{r}^{n}}{\left\|\boldsymbol{r}^{n}\right\|}$ has at least one accumulation point or equivalently at least one convergent subsequence. We denote by $\overline{\boldsymbol{r}}$ one of its accumulations point. Since $F\left(\left(\boldsymbol{r}^{n}\right)_{n}, \boldsymbol{\rho}^{*}\right) \stackrel{n \rightarrow+\infty}{\longrightarrow} F^{*}$ then this is still true for any subsequence extracted from $\left(\boldsymbol{r}^{n}\right)_{n}$. Consider the subsequence corresponding to the accumulation point $\overline{\boldsymbol{r}}$, then for $y \geq 0$ which refers to the modulus, $F^{*}=\lim _{y \rightarrow+\infty} F\left(y \overline{\boldsymbol{r}}, \boldsymbol{\rho}^{*}\right)$. We define the set of all states $\Omega=\left\{\left(a^{j}, x^{i}\right), j=1, \ldots, 2^{K} i=\right.$ $\left.1, \ldots,\left|I_{j}\right|\right\}$, let $m=\max _{S \in \Omega}\left\{\left\langle x^{i}, \overline{\boldsymbol{r}}\right\rangle\right\}$ and denote $\Gamma=\{S \in$ $\left.\Omega:\left\langle x^{i}, \overline{\boldsymbol{r}}\right\rangle=m\right\}$ where $\langle\cdot, \cdot\rangle$ refers to the scalar product. Then as $n_{k} \rightarrow+\infty$, the stationary distribution for a random $S=\left(a^{j}, x^{i}\right) \in \Omega$ of our chain becomes:

$$
\pi\left(a^{j}, x^{i} ; y \overline{\boldsymbol{r}}, \boldsymbol{\rho}^{*}\right)=\frac{\exp \left(\left\langle a^{j}, \boldsymbol{\rho}^{*}\right\rangle\right) \exp \left(\left\langle x^{i}, y \bar{r}\right\rangle\right)}{\sum_{j=1}^{2^{K}} \sum_{i=1}^{\left|I_{j}\right|} \exp \left(\left\langle a^{j}, \boldsymbol{\rho}^{*}\right\rangle\right) \exp \left(\left\langle x^{i}, y \bar{r}\right\rangle\right)}
$$

By multiplying both the numerator and denominator by the same quantity $\exp (-y m)$, it leads to:

$$
\frac{\exp \left(y\left(\left\langle x^{i}, \overline{\boldsymbol{r}}\right\rangle-m+\left\langle a^{j}, \boldsymbol{\rho}^{*}\right\rangle / y\right)\right)}{\sum_{j=1}^{2^{K}} \sum_{i=1}^{\left|I_{j}\right|} \exp \left(y\left(\left\langle x^{i}, \overline{\boldsymbol{r}}\right\rangle-m+\left\langle a^{j}, \boldsymbol{\rho}^{*}\right\rangle / y\right)\right)} \stackrel{y \rightarrow+\infty}{\longrightarrow} \frac{\mathbb{1}\{S \in \Gamma\}}{|\Gamma|}
$$

Since $\left\langle a^{j}, \boldsymbol{\rho}^{*}\right\rangle / y \stackrel{y \rightarrow+\infty}{\longrightarrow} 0$, we are left with the factor $\left\langle x^{i}, \bar{r}\right\rangle-m \leqslant 0$ with y tending to infinity. In order to have a non zero numerator we need that factor to be null or in other words $S \in \Gamma$.

We can distinguish two cases:

- $\Gamma=\Omega$ : In this case, all the states share the same maximum value $m$ hence the limiting distribution is simply the uniform distribution over all the state space. In this case, $\pi\left(a^{j}, x^{i} ; y \overline{\boldsymbol{r}}, \boldsymbol{\rho}^{*}\right)=\pi\left(a^{j}, x^{i}, 0,0\right)$ and $F^{*}=F(0,0)$. Therefore, $\mathrm{F}$ has a finite minimizer $(0,0)$ which contradicts our assumption

- $\Gamma \neq \Omega$ : In this case, there exist at least a state $S^{\prime}=$ $\left(a^{j^{\prime}}, x^{i^{\prime}}\right) \notin \Gamma$ such that $\pi\left(a^{j^{\prime}}, x^{i^{\prime}} ; y \overline{\boldsymbol{r}}, \boldsymbol{\rho}^{*}\right) \stackrel{y \rightarrow+\infty}{\longrightarrow} 0$. However, we assumed that $p_{i^{\prime} j^{\prime}}>0$ and therefore we can conclude, from the expression of the objective function, that $F^{*}=+\infty$ which is clearly not minimal since $F(0,0)<+\infty$. This means that case (b) cannot occur

The proof that case (c) cannot occur is identical to the preceding one but by taking the sequence $\left(\rho^{n}\right)_{n}$ into account instead. The same reasoning can be applied to prove the impossibility of case (d). The two sequences are taken simultaneously and we extract from each a subsequence with their corresponding 
accumulation points. We will have to define the following quantities:

- $m_{1}=\max _{S \in \Omega}\left\{\left\langle a^{j}, \overline{\boldsymbol{\rho}}\right\rangle\right\}$ and denote $\Gamma_{1}=\left\{S \in \Omega:\left\langle a^{j}, \overline{\boldsymbol{\rho}}\right\rangle=\right.$ $\left.m_{1}\right\}$

- $m_{2}=\max _{S \in \Omega}\left\{\left\langle x^{i}, \overline{\boldsymbol{r}}\right\rangle\right\}$ and denote $\Gamma_{2}=\left\{S \in \Omega:\left\langle x^{i}, \overline{\boldsymbol{r}}\right\rangle=\right.$ $\left.m_{2}\right\}$

By multiplying the stationary distribution by $\exp \left(-m_{1} y_{1}-\right.$ $\left.m_{2} y_{2}\right)$, we will end up with:

$$
\pi\left(a^{j}, x^{i} ; y_{2} \overline{\boldsymbol{r}}, y_{1} \overline{\boldsymbol{\rho}}\right) \stackrel{y_{1}, y_{2} \rightarrow+\infty}{\longrightarrow} \frac{\mathbb{1}\left\{S \in \Gamma_{1} \cap \Gamma_{2}\right\}}{\left|\Gamma_{1} \cap \Gamma_{2}\right|}
$$

By following the same analysis as before, we will get to the conclusion that case (d) cannot occur and we are left with case (a) which proves the existence of a unique minimizer $\left(r^{*}, \rho^{*}\right)$ as long as $p_{i j}>0 \forall S \in \Omega$ which brings us back to our original assumptions that it is enough to have $\boldsymbol{\lambda} \in \operatorname{int}(\Lambda)$ and $\boldsymbol{f} \in \operatorname{int}(\Theta(\boldsymbol{\lambda}))$ to have a unique finite minimizer $\left(r^{*}, \rho^{*}\right)$. 